\title{
Difference Forms
}

\author{
Elizabeth L. Mansfield \\ Institute of Mathematics, Statistics and Actuarial Science, \\ University of Kent, Canterbury, CT2 7NF, U.K. \\ E-mail: E.L.Mansfield@kent.ac.uk \\ Peter E. Hydon \\ Department of Mathematics and Statistics, \\ University of Surrey, \\ Guildford GU2 7XH, England. \\ E-mail: P.Hydon@surrey.ac.uk
}

October 17, 2007

\begin{abstract}
Currently, there is much interest in the development of geometric integrators, which retain analogues of geometric properties of an approximated system. This paper provides a means of ensuring that finite difference schemes accurately mirror global properties of approximated systems. To this end, we introduce a cohomology theory for lattice varieties, on which finite difference schemes and other difference equations are defined. We do not assume that there is any continuous space, or that a scheme or difference equation has a continuum limit. This distinguishes our approach from theories of "discrete differential forms" built on simplicial approximations and Whitney forms, and from cohomology theories built on cubical complexes. Indeed, whereas cochains on cubical complexes can be mapped injectively to our difference forms, a bijection may not exist. Thus our approach generalizes what can be achieved with cubical cohomology. The fundamental property that we use to prove our results is the natural ordering on the integers. We show that our cohomology can be calculated from a good cover, just as de Rham cohomology can. We postulate that the dimension of solution space of a globally defined linear recurrence relation equals the analogue of the Euler characteristic for the
\end{abstract}


lattice variety. Most of our exposition deals with forward differences, but little modification is needed to treat other finite difference schemes, including Gauss-Legendre and Preissmann schemes.

\section{Contents}

1 Introduction $\quad 3$

2 Difference forms on lattice varieties $\quad 5$

2.1 Difference forms and the difference map . . . . . . . . . . . . . . 7

2.2 Lattice varieties and difference chains . . . . . . . . . . . . . 8

2.2.1 Fundamental cubes ................... . . . 9

2.2 .2 Difference chains . . . . . . . . . . . . . . . . . . 10

2.2 .3 Lattice varieties . . . . . . . . . . . . . . . . . 13

2.3 The difference complex . . . . . . . . . . . . . . . 17

3 Local exactness $\quad 19$

3.1 Homotopy operators ...................... 19

4 From local to global $\quad 23$

4.1 The Čech difference operator . . . . . . . . . . . . . . . . 23

4.2 Čech cochains . . . . . . . . . . . . . . . . 26

4.3 The Čech- $\Delta$ double complex . . . . . . . . . . . . . . . . 28

5 Examples $\quad 30$

5.1 The punctured plane .................... 30

5.2 An annular lattice . . . . . . . . . . . . . . . . . . . . . . . . . . . . . . . . . 32

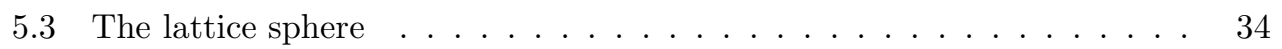

6 Discussion, conjectures and open problems $\quad 37$

6.1 Comparison with cubical cochain theories . . . . . . . . . . . . 37

6.2 Conjectures concerning discrete index theorems . . . . . . . . . . . 38

6.3 What is special about the south west? . . . . . . . . . . . . 40

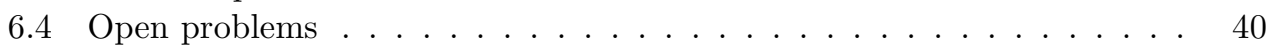

6.4.1 Independence from the good cover . . . . . . . . . . . . . . 40

6.4.2 Non-orientable lattice varieties . . . . . . . . . . . . . . . . . 41

6.4.3 Localized refinements ..................... 41

6.4.4 Functorial properties .................. 41

$\begin{array}{lll}7 & \text { Conclusions } & 41\end{array}$ 


\section{Introduction}

The new discipline of geometric integration focuses on the problem of numerically approximating differential equations while preserving important geometrical structures $[\mathbf{7}, \mathbf{1 5}]$. Integration schemes have been devised that preserve symplectic and multisymplectic structures $[\mathbf{1 2}, \mathbf{3 0}, \mathbf{3 1}]$, variational structures, $[\mathbf{2 2}]$, symmetries $[\mathbf{6}]$, first integrals $[\mathbf{2 5}]$ and conservation laws $[\mathbf{1 4}, \mathbf{2 6}]$. In some instances, it is possible to construct approximations on a Lie group directly $[\mathbf{2 0}]$.

So far, most research in geometric integration has concentrated on the preservation of local structures. However, in many applications, it is also essential to preserve global structures. For smooth manifolds, the cohomology of the de Rham complex can be calculated by well-known techniques. There is much interest in the question of whether analogues of differential forms exist for spaces that are used in numerical methods, for such an analogue could produce a test for ensuring that cohomology is preserved by a numerical approximation.

Cohomology is important to numerical approximation because it encodes topological information, such as topological invariants, obstructions and monodromy. For instance, the automatic calculation of topological properties of a mesh in the context of finite element PDE solvers has been considered [33]. As an example, consider the shallow water equations in computational meteorology, which have an invariant called potential vorticity. The evolution of the distribution of potential vorticity over a domain $M$ largely determines the motion of large-scale weather systems. This is encoded in the potential vorticity 2 -form

$$
\Omega=\mathrm{d} u \wedge \mathrm{d} x+\mathrm{d} v \wedge \mathrm{d} y+f \mathrm{~d} x \wedge \mathrm{d} y
$$

where $(u, v)$ is velocity at position $(x, y)$ and $f$ is the Coriolis parameter, which is nonzero away from the equator. The topology of the domain $M$ constrains the total potential vorticity. For instance, if doubly-periodic boundary conditions are imposed, then the total potential vorticity,

$$
Q:=\int_{M} \Omega
$$

is nonzero. This is because $M$ is topologically equivalent to a 2-torus; the area 2 -form $\mathrm{d} x \wedge \mathrm{d} y$ is closed but not exact. For the same reason, the total potential vorticity $Q$ on a sphere is nonzero [29], whereas $Q$ can be zero on an annulus or a starshaped domain. This illustrates the necessity of preserving the cohomology of the original system in any numerical approximation.

Considerable effort has been focused on analogues of differential forms for computational electromagnetism using the finite element method $[\mathbf{1}, \mathbf{3}, \mathbf{4}, \mathbf{1 3}, \mathbf{1 6}]$; see $[\mathbf{2}]$ for a comprehensive discussion of recent results. These involve an analogue to the de Rham complex that is based on Whitney forms [37] rather than smooth differential forms. A related approach to the general finite element method uses discrete differential forms $[\mathbf{2 4}, \mathbf{3 4}]$ which are actually classical simplicial cochains, that is, linear maps 
from the vertices, edges, faces, and so on, to the coefficient field. The de Rham map yields a classical cochain from a smooth differential form by evaluation or integration over the vertices, edges and so on. Whitney forms are piecewise linear differential forms that are interpolations of classical cochains. If $\omega$ is a differential form, $R$ is the de Rham map and $W$ is the Whitney map, then there is a well-defined sense in which $W R \omega$ is homotopic to $\omega$; this allows the order of the approximation to be quantified.

It is essential to realize that the term 'discrete differential form' does not imply that the underlying space is discrete. Indeed, the finite element method uses a simplicial approximation to a smooth manifold; the space remains continuous even though differentiability is lost at boundaries of simplices.

By contrast, finite difference methods are defined in terms of mesh points, without there being a need for an underlying continuous space. The same is true for difference equations in general; where such equations are used to model an inherently discrete process, the imposition of a continuous structure can produce artefacts. A related philosophical problem was raised by Chard and Shapiro [8], who write of the loss of physical content by the standard process of taking a continuum limit of the discrete process and then re-discretizing for the purpose of computation. Further, there is interest in studying physical systems on discrete space-times (for example, see [38]), particularly in the context of quantum gravity.

Therefore, to deal with difference equations in a theory that uses only concepts germane to difference equations themselves, it is necessary to discard the continuous base space. At first sight this might seem to be disastrous, for most of the familiar and useful constructions are lost. These include continuity (at least in the usual sense), the tangent bundle and the exterior derivative; indeed, difference operators are not derivations, that is, they have no Leibniz product rule. Moreover, loss of continuity means that one must abandon results for constructions that are based on an underlying continuum, such as cohomology theories based on cubical simplices $[\mathbf{2 1}, \mathbf{2 7}]$. In fact, classical cubical chains can be mapped injectively but not bijectively to their difference analogues (see §6.1). Consequently, although some theorems from cubical simplex theories can be adapted to the context of difference equations, they must be reproved without reference to a continuous space (see Remark 17 of $\S 2.2$ ).

As there exist applications which are both inherently discrete and for which there are either multiple or no continuum limits, we prove results for the cohomology theory presented here using only those tools and constructions which pertain to difference equations themselves, such as the shift operator. We show that by exploiting a single property of difference equations it is possible to derive analogues of several major results concerning the de Rham complex. In finite difference methods, mesh points do not need to be evenly spaced [6], but they are ordered in each direction by an integer label, at least locally. Instead of dealing with mesh points directly, it is helpful to regard the independent variables (coordinates) as being $p$-tuples of integers, where $p$ is the dimension of the discretized problem. The usual ordering on $\mathbb{Z}$ provides sufficient structure, namely adjacency and orientation, to enable us to derive difference analogues of chains (§1), exterior algebra, a coboundary operator, the de Rham complex and 
Stokes' Theorem ( $\S 2)$; each analogue is adapted to a particular finite difference method. In $\S 3$, we construct a homotopy operator to prove that the difference complex is locally exact. This provides an analogue of the existence of local potentials for curl-free and divergence-free fields in the smooth theory.

In the continuous case, one can regard a manifold as the result of gluing together coordinate patches. Similarly, it is possible to glue together pieces of mesh, each of which has its own coordinate system as above, to form what we call a lattice variety. In $\S 4$, we combine the difference complex with a Čech complex, enabling the cohomology of a given lattice variety to be calculated. Several examples are presented in $\S 5$ and the paper concludes with some conjectures and open problems $(\S 6)$. In particular, we give evidence for a conjecture that the dimension of the solution space of a globally defined linear recurrence relation equals the analogue of the Euler characteristic for the lattice variety. As the same quantity appears in the Morse Index Theorem, our conjecture might be thought to be (in some distant sense) an analogue of the Morse Index theorem for difference systems on lattice varieties. However, our difference index is not a result of a discrete Morse theory, such as the one constructed by Forman [9].

\section{Difference forms on lattice varieties}

The simplest $p$-dimensional lattice is $\mathbb{Z}^{p}$, whose points are identified by the labels

$$
\mathbf{n}=\left(n^{1}, \ldots, n^{p}\right), \quad n^{i} \in \mathbb{Z}, \quad i=1, \ldots, p .
$$

These labels provide a coordinate system; points are ordered in the $i^{\text {th }}$ direction by the coordinate $n^{i}$. Henceforth, in each of our two-dimensional examples, we shall use $n^{1}=m$ as the coordinate in the horizontal direction and $n^{2}=n$ as the coordinate in the vertical direction.

The natural ordering in $\mathbb{Z}$ yields the notions of adjacency and orientation, as well as the notions of both forward and backward differences. The most important of these in the construction of a lattice variety is adjacency.

Definition 1 Two points with coordinates $\mathbf{m}=\left(m^{1}, \ldots, m^{p}\right)$ and $\mathbf{n}=\left(n^{1}, \ldots, n^{p}\right)$ in $\mathbb{Z}^{p}$ are said to be adjacent if and only if

$$
|\mathbf{m}-\mathbf{n}|:=\sum_{i=1}^{p}\left|m^{i}-n^{i}\right|=1 .
$$

Remark 2 In the digital topology literature, adjacency is called '4-connectedness' when $p=2$; the notion of '8-connectedness' is not needed here, see for example [23].

Definition $3 A$ lattice $L$ is any subset of $\mathbb{Z}^{p}$ with the property that every point is adjacent to at least one other point.

The role of adjacency is similar to that of connectedness for manifolds. Consequently, the only allowable coordinate changes on $L$ are those that preserve adjacency, namely translations, rotations by a multiple of $\pi / 4$ and reflections. 
Definition 4 A path in a lattice $L$ from $\mathbf{n}_{0}$ to $\mathbf{n}_{1}$ is an ordered set $P$ of points in $L$, such that

1. the first point in $P$ is $\mathbf{n}_{0}$;

2. the last point in $P$ is $\mathbf{n}_{1}$;

3. every other point in $P$ is adjacent to its two immediate neighbours in $P$.

Example 5 Each of the following ordered sets is a path in $\mathbb{Z}^{2}$ from $(3,3)$ to $(5,4)$ :

$$
\begin{aligned}
& P_{1}=((3,3),(3,4),(4,4),(4,3),(5,3),(5,4)) \\
& P_{2}=((3,3),(4,3),(5,3),(5,4)) \\
& P_{3}=((3,3),(3,4),(4,4),(5,4)) .
\end{aligned}
$$

Definition 6 Given a lattice $L \subset \mathbb{Z}^{p}$ and a permutation $\pi$ of the indices $1, \ldots p$, a $\pi$-ordered path is a path in $L$ for which all changes to the $\pi(i)^{\text {th }}$ coordinate (as one moves along the path) occur before any changes to the $\pi(i+1)^{\text {th }}$ coordinate (for each $i$ in turn). Hence the $\pi(1)^{\text {th }}$ coordinate reaches its final value before any change is made to the $\pi(2)^{\text {th }}$ coordinate, and so on.

For a particular permutation $\pi$, a $\pi$-ordered path between two given points may not exist, because $L$ might lack some of the necessary intermediate points; however, if a $\pi$-ordered path exists, it is unique. In Example 5, the paths $P_{2}$ and $P_{3}$ from $(3,3)$ to $(5,4)$ are the $(1,2)$-ordered and $(2,1)$-ordered paths in $\mathbb{Z}^{2}$, respectively. Our motivation for identifying $\pi$-ordered paths will be seen in $\S 3$, where we prove an analogue of the Poincaré Lemma by constructing a homotopy operator that uses projection along such paths. The idea of $\pi$-ordered paths is tied to the coordinate system that is used, but the following definition is independent of the choice of coordinates.

Definition 7 A lattice $L \subset \mathbb{Z}^{p}$ is projectable if there exists a point $\mathbf{n}_{0} \in L$ and a permutation $\pi$ such that, for each $\mathbf{n} \in L$, there is a $\pi$-ordered path from $\mathbf{n}$ to $\mathbf{n}_{0}$.

In the diagram below, we show three examples of lattices in $\mathbb{Z}^{2}$. Only the one on the left is projectable. In this instance, we can choose $\mathbf{n}_{0}$ to be the point marked by a diamond; there is a path from every other point $\mathbf{n} \in L$ to this point such that the changes in horizontal coordinate precede changes in the vertical coordinate. The other two lattices are not projectable: they have no point that can be reached from all other points along a $\pi$-ordered path (for any fixed permutation $\pi$ ).
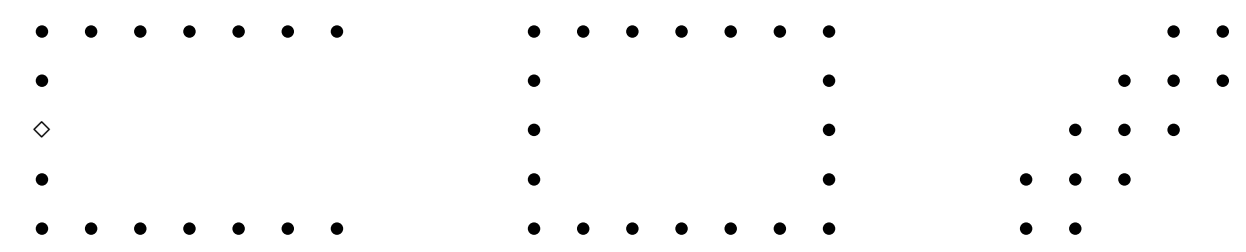
From an algebraic viewpoint, moving between adjacent points amounts to shifting one of the coordinates by \pm 1 , leaving the other coordinates unchanged. This leads to the idea of shift maps, which act on objects that are defined on a given lattice $L$.

Definition 8 For each $k \in\{1, \ldots, p\}$, the $k^{\text {th }}$ shift map is defined by:

$$
S_{k}:\left\{\begin{aligned}
n^{i} & \mapsto n^{i}+\delta_{k}^{i} ; \\
\mathbf{n} & \mapsto \mathbf{n}+\mathbf{1}_{k} .
\end{aligned}\right.
$$

where $\mathbf{1}_{k}$ is the p-tuple whose only nonzero entry is the $k^{\text {th }}$, which is 1 , and where $n^{i}$ is defined in equation (1).

Let $\mathcal{B}$ denote the set of all real-valued (alternatively, complex-valued) functions defined on $\mathbb{Z}^{p}$. The shift maps have the following properties:

1. $S_{j} S_{k}=S_{k} S_{j}$

2. $S_{k} f(\mathbf{n})=f\left(\mathbf{n}+\mathbf{1}_{k}\right)$, for all $f \in \mathcal{B}$;

3. $S_{k}(f(\mathbf{n}) g(\mathbf{n}))=S_{k}(f(\mathbf{n})) S_{k}(g(\mathbf{n}))$ for all $f, g \in \mathcal{B}$.

These properties also hold on any lattice $L$, except that shift maps and their inverses can only act in directions for which the shifted point belongs to $L$ (otherwise, they are undefined).

The standard (positive) orientation on $\mathbb{Z}^{p}$ is defined analogously to that for $\mathbb{R}^{p}$, where it can be defined in terms of an ordered sequence of the standard basis vectors. The positive orientation can be represented by the $(1, \ldots, p)$-ordered path $O\left(\mathbf{n}_{0}\right)$ from a given point $\mathbf{n}_{0}$ to the point $\mathbf{n}_{0}+\mathbf{1}_{1}+\cdots+\mathbf{1}_{p}$. For instance, when $p=2$ this path is

$$
O\left(m_{0}, n_{0}\right)=\left(\left(m_{0}, n_{0}\right),\left(m_{0}+1, n_{0}\right),\left(m_{0}+1, n_{0}+1\right)\right) .
$$

An orientation defined by any other $\pi$-ordered path is then determined by the parity of the permutation $\pi$, namely negative for odd permutations and positive for even. There is a naturally induced orientation on the cubes comprising the boundary by taking the relevant subpath. The standard orientation on any lattice $L \subset \mathbb{Z}^{p}$ is obtained in the same way. In our examples, we shall use arrows to indicate this orientation when it is of particular importance.

\subsection{Difference forms and the difference map}

Let $\mathbf{E x}(p)$ be the exterior algebra on $p$ symbols, $\Delta_{1}, \ldots, \Delta_{p}$, with real (or complex) coefficients, so that

$$
\Delta_{i} \wedge \Delta_{i}=0, \quad \Delta_{i} \wedge \Delta_{j}=-\Delta_{i} \wedge \Delta_{j} .
$$

Define the algebra of difference forms to be

$$
{ }^{p} \mathbf{E x}=\left.\bigcup_{\mathbf{n} \in \mathbb{Z}^{p}} \mathbf{E x}(p)\right|_{\mathbf{n}},
$$


with coefficients in $\mathcal{B}$. Difference $r$-forms on $\mathbb{Z}^{p}$ are written as $\omega \in{ }^{p} \mathbf{E x}^{r}$. [Whenever it is necessary to restrict attention to a particular lattice $L \subset \mathbb{Z}^{p}$ we write $\omega \in{ }^{p} \mathbf{E x}^{r}(L)$.] By analogy with differential forms,

$$
\omega= \begin{cases}\sum_{i_{1}<\cdots<i_{r}} f_{i_{1} \cdots i_{r}}(\mathbf{n}) \Delta_{i_{1}} \wedge \Delta_{i_{2}} \wedge \cdots \wedge \Delta_{i_{r}}, & r \geq 1 \\ f_{0}(\mathbf{n}), & r=0 .\end{cases}
$$

Here $f_{0}, f_{i_{1} \cdots i_{r}} \in \mathcal{B}$. The action of the $k^{\text {th }}$ shift map on difference forms is defined by

$$
\begin{aligned}
& S_{k}\left(\Delta_{i}\right)=\Delta_{i} \\
& S_{k}(\eta \wedge \omega)=S_{k}(\eta) \wedge S_{k}(\omega), \quad \eta, \omega \in{ }^{p} \mathbf{E x} .
\end{aligned}
$$

Definition 9 The difference map $\boldsymbol{\Delta}:{ }^{p} \mathbf{E} \mathbf{x}^{r} \rightarrow{ }^{p} \mathbf{E} \mathbf{x}^{r+1}$ is defined by

$$
\boldsymbol{\Delta}(\omega)=\sum_{k=1}^{p} \Delta_{k} \wedge\left(S_{k}-i d\right) \omega .
$$

Note that this map uses forward differences, whereas most finite difference methods do not. For simplicity, attention is restricted to forward differences until $\S 6$, where we briefly mention some minor changes that enable difference forms to be used far more widely, for example with backward difference and collocation methods.

The difference map plays the role of the exterior derivative; it has the analogous properties

i) $\boldsymbol{\Delta}^{2}=0$

ii) $\boldsymbol{\Delta}\left(n^{i}\right)=\Delta_{i}$.

However, unlike the exterior derivative, the difference map is not a derivation:

$$
\boldsymbol{\Delta}(\eta \wedge \omega) \neq \boldsymbol{\Delta}(\eta) \wedge \omega \pm \eta \wedge \boldsymbol{\Delta}(\omega) .
$$

The analogue of the de Rham complex, which we call the difference complex, will be defined in $\S 2.3$.

\subsection{Lattice varieties and difference chains}

Earlier, we introduced the idea of lattice varieties, which are the natural analogues of manifolds. A manifold is a topological space that can be covered by coordinate charts, subject to certain conditions (continuity, smoothness, etc.) where charts overlap. For lattice varieties, continuity is replaced by adjacency. Thus it is natural to adopt the following working definition of a lattice variety.

Definition 10 A p-dimensional lattice variety $L$ is a set of points that can be covered by subsets $L_{\alpha}$, each of which is mapped by a injection $\phi_{\alpha}$ to a subset of $\mathbb{Z}^{p}$ that is a projectable lattice. Furthermore, on any overlap $L_{\alpha} \cap L_{\beta}$, the gluing map $\phi_{\alpha} \circ \phi_{\beta}^{-1}$ must preserve adjacency. 
In $\S 2.2 .3$, we develop a more detailed definition. However, it is helpful to begin by looking at fundamental cubes $(\S 2.2 .1)$, from which all lattice varieties can be constructed. From the set-theoretic viewpoint, every lattice is a union of fundamental cubes. The natural algebraic extension of this idea is that of difference chains, which are formal sums of fundamental cubes. A lattice, as a union of cubes, is regarded as equivalent to a formal sum of the cubes with coefficients either \pm 1 , according to their orientation. In $\S 2.2 .2$, we use difference chains to state a discrete analogue of Stokes' Theorem.

\subsubsection{Fundamental cubes}

A fundamental $p$-cube is a unit cube in $\mathbb{Z}^{p}$, together with information concerning what kinds of forms at each point. The fundamental cubes in dimensions 0,1 and 2 are as follows. Usually we shall use a suffix $\sigma$ as an index that identifies a particular copy of the fundamental cube. Here, for clarity, we use coordinates $(m, n)$ as indices.

$$
\boldsymbol{p}=\mathbf{0} \quad C^{0}=
$$

A single point; only 0 -forms can be defined here.

$$
\boldsymbol{p}=\mathbf{1} \quad C_{m}^{1}=\underset{m}{\longrightarrow} \mathbf{\square}_{m+1}
$$

The forward difference of a function is defined only at $m$, so 0 -forms and 1-forms are definable there. The arrow indicates the orientation.
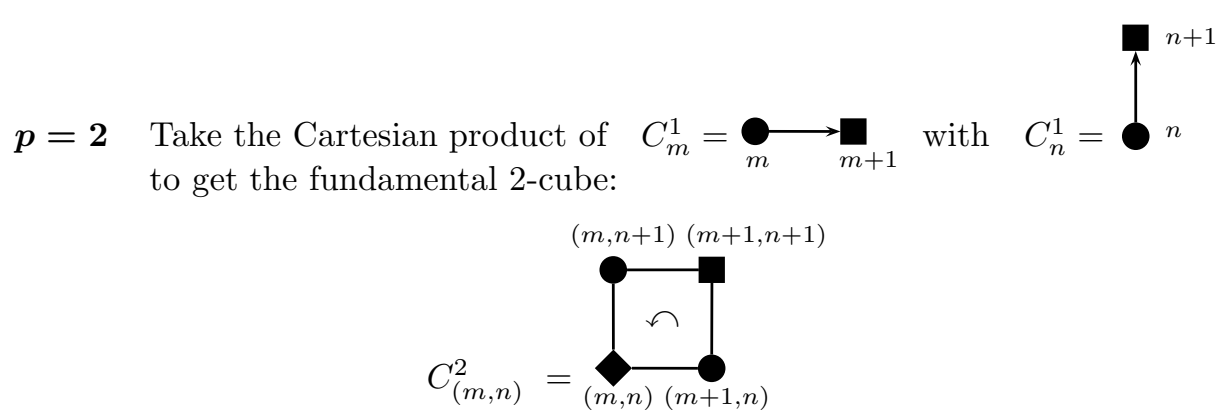

where the orientation is positive, i. e. anticlockwise. (If the coordinates had been ordered $(n, m)$, the orientation would have been reversed.) The diamond at $(m, n)$ indicates that $r$-forms may be defined there for $r \leq 2$.

From here on, we shall state only the maximum order $r_{\max }$ of difference forms that can be defined at a point in a lattice; difference forms of all orders $r<r_{\max }$ can also be defined at that point.

Addition of fundamental cubes (and, more generally, difference chains) is defined in exactly the same way as the addition of chains is in the continuous case. As usual, a minus sign is used when the orientation is reversed. 
Key Rule: If a difference form can be defined at a point, it remains defined there when other fundamental cubes are added.

For example,

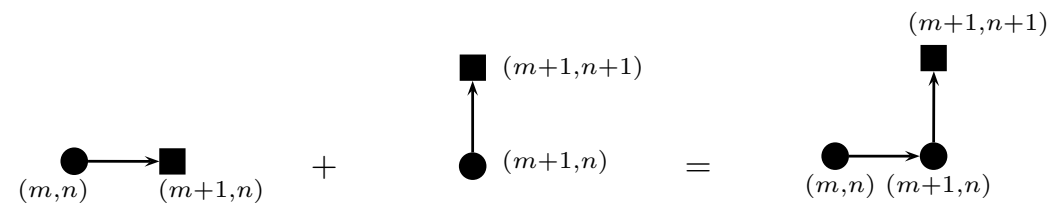

(ii)

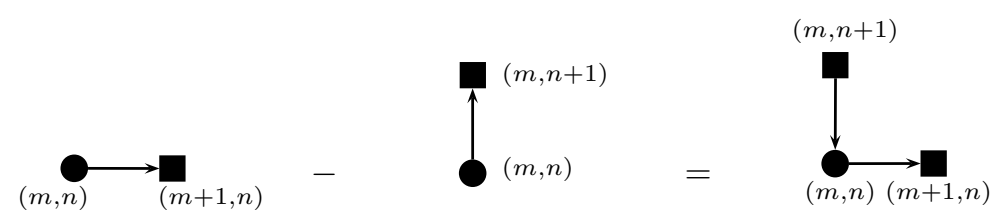

In example (ii) above, there is no diamond at $(m, n)$ because the square is incomplete; even though the forward difference can be taken in each direction, 2-forms cannot properly be defined there and so the set of 2 -forms at such a point is $\{0\}$. Thus, the action of $\boldsymbol{\Delta}$ on a 1 -form at $(m, n)$ is zero for this lattice.

Definition 11 The corner of a fundamental p-cube at which p-forms can be defined will be denoted the south west corner. The remaining points will be denoted collectively as TopRight points.

(If we had used backward differences, $p$-forms would only be definable at the north east corner; all other points would be referred to as BottomLeft points. We use a geographical description only for points at which all $r$-forms $(r \leq p)$ can be defined.) The above definition will later be extended to lattice varieties (see Definition 21). The TopRight points turn out to have an important role in the proofs in $\S 4$.

Figure 1 shows a lattice with two points removed. Its component fundamental cubes are easily identified, bearing in mind the Key Rule above. The only points at which 2 -forms can be defined are marked by diamonds; all other points are TopRight points.

\subsubsection{Difference chains}

A useful algebraic approach extends addition of fundamental cubes to include all integer coefficients. This is the natural way to prove the analogue of Stokes' Theorem, which we state in this section.

Definition $12 A$ difference $p$-chain is a formal linear sum of fundamental p-cubes with integer coefficients.

The boundary of a difference $p$-chain $C^{p}$ is denoted by $\partial C^{p}$. The boundary operator $\partial$ is a linear operator that maps $p$-chains to $(p-1)$-chains. In particular, the boundary 
Figure 1: Lattice with two points removed

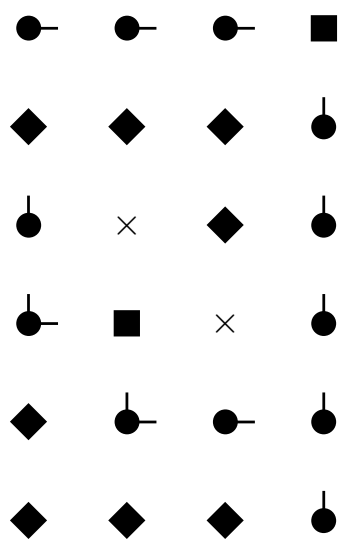

Key: — one form defined in direction indicated

- zero form two form defined

$\times$ point removed

of a fundamental cube $C^{p}$ is defined recursively as follows. Regard $C^{p}$ as $C^{p-1} \times C^{1}$; then

$$
\partial C^{p}=\left(\partial C^{p-1}\right) \times C^{1}+(-1)^{p-1} C^{p-1} \times \partial C^{1} .
$$

(A non-recursive formula given by Fulton ( $[\mathbf{1 1}]$, p. 333) is also easily adapted to the discrete case.) The recursive method of calculating the boundary yields the following results for the fundamental cubes that are listed in $\S 2.2 .1$ :

$$
\begin{aligned}
& \partial C_{\sigma}^{0}=0 \\
& \partial C_{\sigma}^{1}=\underset{m+1}{\mathbf{q}}-\mathbf{\square}_{m} \\
& \partial C_{\sigma}^{2}=\underset{(m, n)}{\longrightarrow} \boldsymbol{q}_{(m+1, n)}+\oint_{(m+1, n)}^{(m+1, n+1)} \\
& -\overbrace{(m, n+1)}^{\longrightarrow} \underbrace{(m, n+1)}_{(m+1, n+1)}
\end{aligned}
$$

Note that

$$
\left.\partial\left(\partial C_{\sigma}^{2}\right)\right)=0
$$


For a general difference $p$-chain, the boundary can be calculated by summing the boundaries of the component fundamental $p$-cubes. For any $p$-chain that is an oriented lattice regarded as a union of fundamental $p$-cubes each of which has the positive orientation, and is thus represented as a sum of the component cubes with coefficients all equal to +1 , the internal boundaries cancel out. (Each internal boundary is common to two of the $p$-cubes; each is counted once with the coefficient +1 and once with the coefficient -1 .)

To state Stokes' Theorem, all that remains is to define the difference analogue of the integral of an $r$-form over an $r$-chain.

Definition 13 Given an r-chain $C^{r}$,

$$
C^{r}=\sum_{\sigma} a_{\sigma} C_{\sigma}^{r}, \quad a_{\sigma} \in \mathbb{Z},
$$

and an $r$-form of the form

$$
\omega=F\left(n^{1}, \ldots, n^{r}\right) \Delta_{1} \wedge \cdots \wedge \Delta_{r}
$$

the oriented sum of $\omega$ over $C^{r}$ is defined to be

$$
\sum_{C^{r}} \omega=\sum_{\sigma} a_{\sigma} F\left(n^{1}(\sigma), \ldots, n^{r}(\sigma)\right)
$$

provided that $\omega$ is defined at $\left(n^{1}(\sigma), \ldots, n^{r}(\sigma)\right)$. This definition is extended to arbitrary $r$-forms by linearity.

For example,

\begin{tabular}{llll}
$p$ & $C^{p}$ & $\omega$ & $\sum_{C^{p}} \omega$ \\
\hline 0 & $a_{a}$ & $F$ & $F(a)$ \\
1 & $\mathrm{a}_{b} \rightarrow{ }_{c}^{\longrightarrow}{ }_{d}$ & $F \Delta_{m}$ & $F(a)+F(b)+F(c)$
\end{tabular}

Theorem: 'Stokes' Theorem for difference forms' Let $C^{p}$ be a finite $p$-chain and let $\omega$ be a $(p-1)$-form. Then

$$
\sum_{C^{p}} \Delta \omega=\sum_{\partial C^{p}} \omega
$$

Consequently $\sum_{\partial C^{p}} \omega=0$ if $\omega$ is closed. The theorem is proved by applying Definition 13 to both sides of the equation. 


\subsubsection{Lattice varieties}

The building blocks of a $p$-dimensional lattice variety $L$ are the fundamental cubes in $\mathbb{Z}^{p}$, which are glued together along boundaries (of dimension $p-1$ ). It is necessary to ensure that adjacency is preserved (together with orientation for an orientable lattice variety). The boundary of $L$ is the sum of the boundaries of the cubes in $L$. For example, the cube corner shown below consists of three two-cells glued together; the diamonds show where two-forms are defined, the square where only a zero-form is defined.

\section{A corner of a cube's surface}

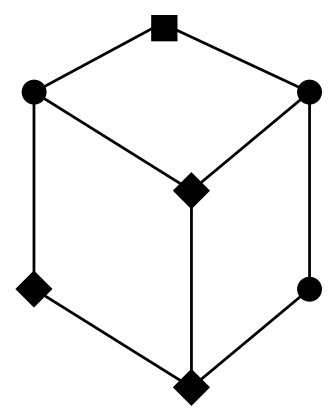

Rather than working solely with fundamental $p$-cubes, it is helpful to glue together larger pieces of lattice. We shall join $p$-dimensional projectable lattices together pairwise, allowing only intersections that respect adjacency and orientation. Each disjoint piece of the intersection is either an overlap (of dimension $p$ ) or part of a boundary (of dimension $p-1)$.

If adjacency is respected but orientation is not, it is possible to construct nonorientable lattice varieties such as a discrete version of the Möbius strip. Except for a brief discussion in $\S 6$, we shall deal only with orientable lattice varieties henceforth. It is possible to respect orientation while violating adjacency (see §6.3.3); in the context of numerical methods, this situation occurs if a mesh is refined locally but not globally. For simplicity, we do not allow this.

The gluing process may be described formally as follows.

Definition 14 A subset $L_{\alpha} \subset L$ and a map

$$
\phi_{\alpha}: \operatorname{supp}(\phi)=L_{\alpha} \mapsto \mathbb{Z}^{p}
$$

is said to be a coordinate chart on $L$ if $\phi$ is injective and $\phi\left(L_{\alpha}\right)$ is a projectable lattice.

Definition 15 Given two such charts on L, we say that the gluing map (or coordinate change map)

$$
\phi_{\alpha} \circ \phi_{\beta}^{-1}: \phi_{\beta}\left(\operatorname{supp}\left(\phi_{\alpha}\right) \cap \operatorname{supp}\left(\phi_{\beta}\right)\right) \rightarrow \mathbb{Z}^{p}
$$


is admissible if for any two points $x_{1}$ and $x_{2}, \phi_{\alpha}\left(x_{1}\right)$ is adjacent to $\phi_{\alpha}\left(x_{2}\right)$ if and only if $\phi_{\beta}\left(x_{1}\right)$ is adjacent to $\phi_{\beta}\left(x_{2}\right)$.

In other words, admissible gluing maps maintain adjacency. However, we need more than a cover of $L$ with admissible gluing maps. Since a variety is constructed out of charts, then for a forward difference to be defined at some point, it must be defined in some $L_{\alpha}$ which contains it. It is not enough that "the dots are all there".

Thus the $\phi_{\alpha}$ also encode which difference forms are defined where in the $L_{\alpha}$. Difference forms can only be calculated in co-ordinates. Thus we take the difference forms on $L_{\alpha}$ to be those highest dimensional forms definable on $\phi_{\alpha}\left(L_{\alpha}\right)$. If a point in $L$ lies in several $L_{\alpha}$, we may take the highest dimensional form that can be defined there. We assume this in the examples that follow.

Example 16 Consider the 2-dimensional lattice variety $L=L_{1} \cup L_{2}$ in Figure 2; its points cover one corner of a $3 \times 3 \times 3$ lattice cube. (We have distorted the cube somewhat, for ease of viewing. Note that although we have shaded the cells in $L_{1}$ to indicate the points that are in $L_{1}$, the 2-dimensional surfaces spanning the vertices are not present in the lattice variety, nor are the edges that are drawn to show the existence of difference 1-forms.) If we take the co-ordinates on $L_{1}$ and $L_{2}$ to be such that their lower left cubes are the unit cube in $\mathbb{Z}^{2}$, that is, 2-forms are defined at their lower left-most points, then a 2-form at $\diamond$ is not defined in either $L_{1}$ or $L_{2}$ and is thus undefined in $L$. Indeed, $L_{1} \cap L_{2}$ has two components. It turns out that the cohomology of this lattice variety is not that of a disc, but that of an annulus. We will see in $\S 6$ that this example illustrates an important difference between lattice varieties and cubical cochains.

Figure 2: Cautionary example 1.

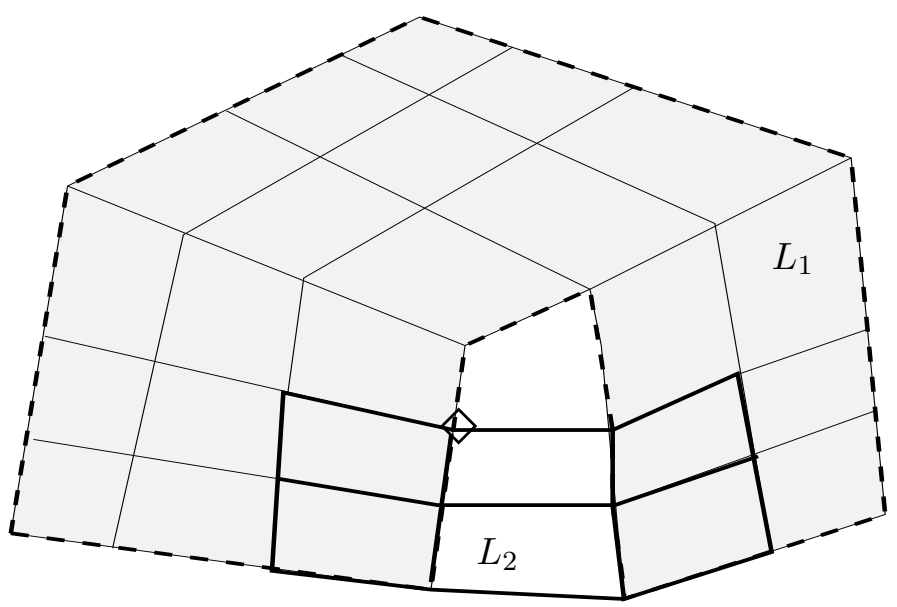

Key: $\diamond$ two-form undefined 
While a lattice variety is constructed from projectable lattices glued together, and these determine what forms are defined where, we need our covers to satisfy a particular property in order to calculate the cohomology of the variety.

Definition 17 We say a cover $\mathcal{L}=\left\{L_{\alpha} \mid \alpha \in A\right\}$ of a $p$-dimensional lattice variety $L$ is valid if for every lattice point $P \in L$ there is an $\alpha=\alpha(P)$ such that any $p$-form definable at $P$ is definable in $L_{\alpha}$.

Example 18 An invalid cover of a 1-cell. The 1-form at the point $P$ is defined in neither $L_{1}$ nor $L_{2}$.

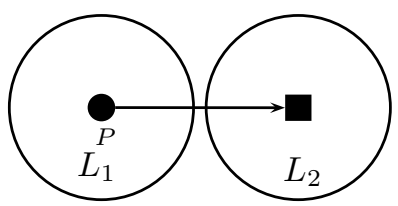

Definition 19 A lattice variety $L$ may be defined as a collection of charts $\left\{\left(L_{\alpha}, \phi_{\alpha}\right)\right\}$ such that $L=\cup_{\alpha} L_{\alpha}$, the cover $\mathcal{L}=\left\{L_{\alpha}\right\}$ is valid, and whenever $\phi_{\alpha} \circ \phi_{\beta}^{-1}$ is defined, then it is admissible.

Example 20 Two lattice varieties modelling the 2-sphere are depicted below. The lines indicate adjacency only; there are no edges or faces in lattice varieties.

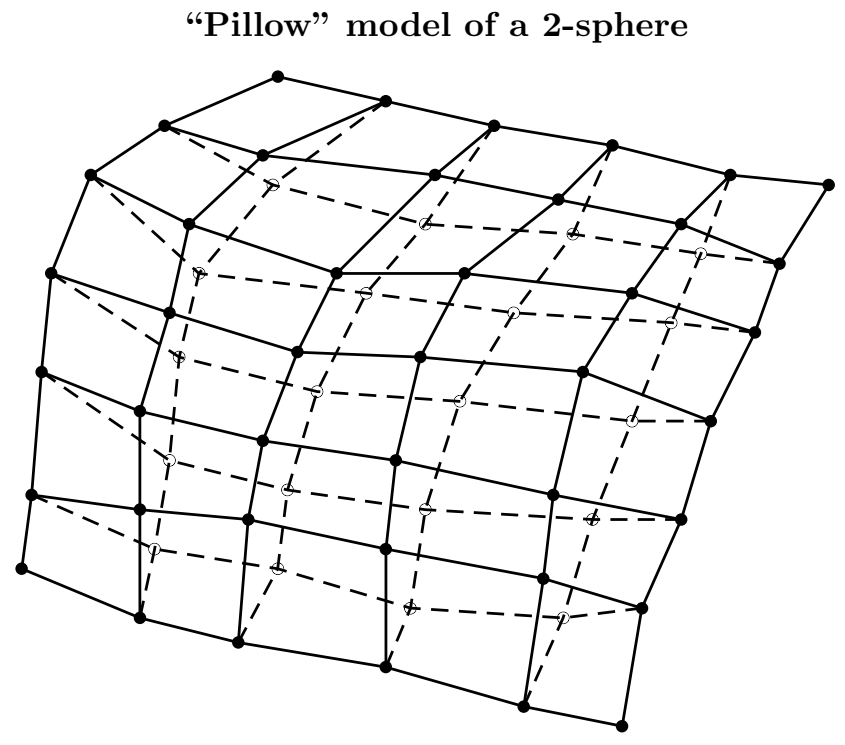




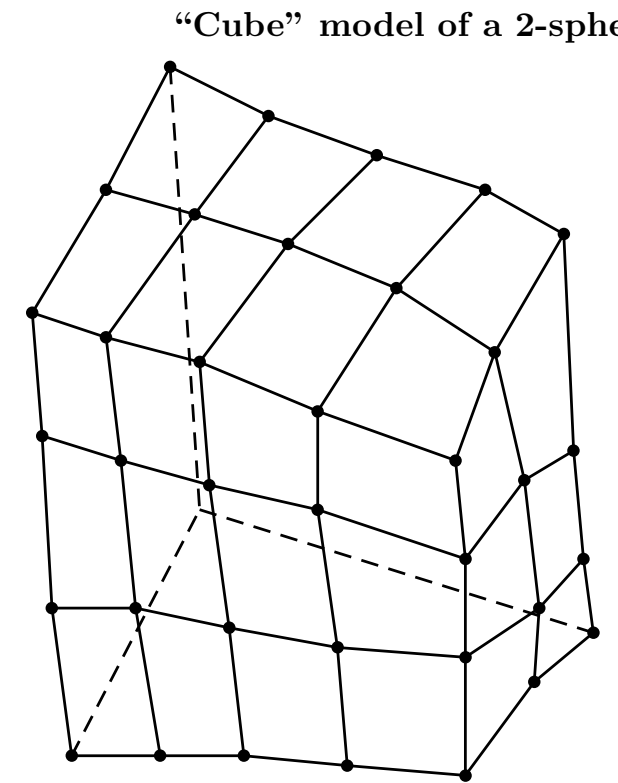

(For simplicity, back faces are not indicated.)

Those points in a lattice variety of dimension $p$ where a $p$-form cannot be defined is an important set. Because domains add when we glue lattices together, we may generalize Definition 11 and define the TopRight of a lattice $L$ as follows.

Definition 21 The TopRight points of a lattice variety of dimension $p$ are those where in no $L_{\alpha}$ containing that point is a p-form defined.

Example 22 For the two-dimensional annular lattice shown below, the TopRight points with respect to the standard chart (given by the obvious inclusion into $\mathbb{Z}^{2}$ ) are marked with a diamond. Changing the chart, for example by rotating the lattice before inclusion, will result in a different set of TopRight points.

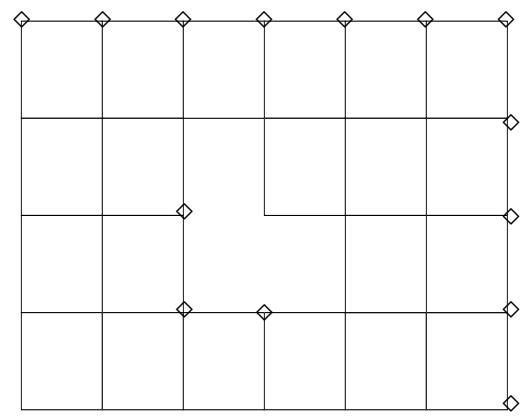


Definition 23 At any point $\mathbf{n} \in L$ where forms of dimension higher than $q$ (say) are not defined, we set

$$
\left.{ }^{p} \mathbf{E x}(L)\right|_{\mathbf{n}} \equiv\{0\}, \quad p \geq q .
$$

Remark 24 There is a strong visual analogy between cubical complexes and lattice varieties (regarded as a sum of fundamental cubes). As the following example shows, the correspondence can only be taken so far.

Example 25 Cautionary example 2. On the left a smooth 2-dimensional cubical cell has its boundary identified to a point * (the equator is for visualization only). The result is $S^{2}$, which is still 2-dimensional. On the right, the four lattice points are identified to a point. The 2-difference form is necessarily lost and the result is zero-dimensional. This example shows that problems occur when there are "not enough dots".

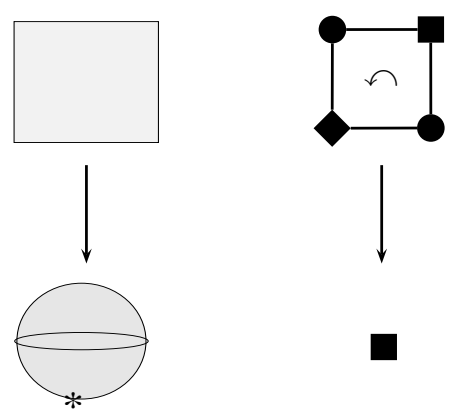

Identification of boundary points for smooth (left) and discrete (right) 2-cells

\subsection{The difference complex}

Difference forms and the difference operator are defined on lattice varieties in the usual way, via the coordinate system provided by the charts. The de Rham complex for differential forms has a difference analogue, which arises from the property $\boldsymbol{\Delta}^{2}=0$.

Definition 26 A difference $r$-form $\omega$ is closed if $\boldsymbol{\Delta}(\omega)=0$ and is exact if there exists $a(r-1)$-form $\eta$ such that $\omega=\boldsymbol{\Delta}(\eta)$. We call $\eta$ a difference potential for $\omega$.

Clearly, every exact $r$-form is closed; we shall prove that the converse is true (for $r \geq 1$ ) on projectable lattices.

Definition 27 The difference complex is

$$
0 \rightarrow \mathbb{R} \rightarrow{ }^{p} \mathbf{E x}^{0} \stackrel{\Delta}{\rightarrow}{ }^{p} \mathbf{x}^{1} \stackrel{\Delta}{\rightarrow} \ldots \stackrel{\Delta}{\rightarrow}{ }^{p} \mathbf{E x}^{p} \stackrel{\Delta}{\rightarrow} 0
$$

For any lattice variety $L$, the cohomology groups are

$$
H_{\Delta}^{r}(L)=\frac{\{\text { closed } r \text {-forms on } L\}}{\{\text { exact } r \text {-forms on } L\}}=\frac{\left.\operatorname{ker} \boldsymbol{\Delta}\right|_{\mathbf{E x}^{r}(L)}}{\left.\operatorname{im} \boldsymbol{\Delta}\right|_{\mathbf{E x}^{r-1}(L)}} .
$$


For $r=0$, the dimension of the group $H_{\Delta}^{0}(L)$ (regarded as a vector space) is the number of distinct connected pieces in $L$. In $\S 3$, we shall prove a generalization of the following result, which was proved in Hydon \& Mansfield (2004).

Theorem For any $p \geq 1$, there exists a homotopy operator $H$ on $\mathbb{Z}^{p}$ such that

$$
H(\boldsymbol{\Delta} \omega)+\boldsymbol{\Delta} H(\omega)=\omega
$$

for every $r$-form $(r \geq 1) \omega$ defined on $\mathbb{Z}^{p}$. Consequently $\omega$ is closed $(\boldsymbol{\Delta} \omega=0)$ if and only if it is exact. Therefore

$$
\begin{aligned}
& H_{\Delta}^{0}\left(\mathbb{Z}^{p}\right) \cong \mathbb{R}, \quad\left(\text { constant functions on } \mathbb{Z}^{p}\right) \\
& H_{\Delta}^{r}\left(\mathbb{Z}^{p}\right) \cong 0, \quad r \geq 1 .
\end{aligned}
$$

Our generalization of the homotopy operator provides one means of constructing the cohomology groups. In $\S 5.1$, we calculate the cohomology groups for the following example.

\section{Example 28 The punctured planar lattice}

For $L=\mathbb{Z}^{2} \backslash\{(0,0)\}$, consider the 1 -forms

$$
\left.\omega_{c}\right|_{(m, n)}=\left\{\begin{array}{cl}
c \Delta_{2}, & \text { if } n=0 \text { and } m \leq-1 \\
0 & \text { otherwise }
\end{array}\right.
$$

where $c$ is constant. In the portion of the lattice $L$ shown, the form equals $c \Delta_{2}$ on the diamonds and is zero on dots. The missing point is marked by $\times$.

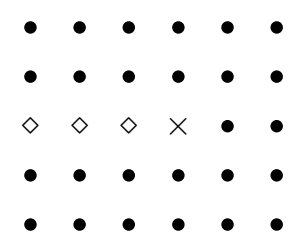

Clearly, $\left(S_{1}-i d\right) \omega_{c}$ is zero wherever it is defined. Thus $\omega_{c}$ is closed, but if $c \neq 0$ then it is not exact. This can be seen by trying to find a pre-image by "integrating" around the hole. In fact,

$$
\begin{aligned}
H_{\Delta}^{1}\left(\mathbb{Z}^{2} \backslash\{(0,0)\}\right) & =\left\{\omega_{c}: c \in \mathbb{R}\right\} \\
& \cong \mathbb{R}
\end{aligned}
$$

so the punctured lattice has cohomology which is isomorphic to the de Rham cohomology of $\mathbb{R}^{2} \backslash\{(0,0)\}$. 


\section{Local exactness}

Local exactness of the de Rham complex is the same as local existence of potentials. In $\mathbb{R}^{3}$, for instance, if a field has zero curl then locally it is a gradient; if it has zero divergence then locally it is a curl. Here we prove local exactness for the difference complex.

For a manifold, the Poincaré Lemma states that every closed $r$-form is exact on a starshaped domain $D$ if $r \geq 1$. The proof uses integration along the ray between a fixed point $\mathbf{x}_{0} \in D$ and an arbitrary point $\mathbf{x} \in D$. For lattice varieties, a different approach is needed, because points on most rays are not adjacent. Instead, a homotopy operator is formed by projecting in each direction in turn, reducing the dimension of the problem by one at each stage. Consequently, the replacement for a starshaped domain is a projectable lattice. The difference analogue of the Poincaré Lemma is as follows.

Lemma 29 The difference complex (7) is exact on every projectable lattice L; hence

$$
\left.\operatorname{ker} \boldsymbol{\Delta}\right|_{p_{\mathbf{E x}^{r}(L)}}=\left.\operatorname{im} \boldsymbol{\Delta}\right|_{p_{\mathbf{E x}^{r-1}(L)}}, \quad r=1, \ldots p-1,
$$

and $\left.\operatorname{ker} \boldsymbol{\Delta}\right|_{{ } \mathbf{E x}^{0}(L)}=\mathbb{R}$.

\subsection{Homotopy operators}

We prove Lemma 29 by constructing a homotopy operator $H:{ }^{p} \mathbf{E}^{r}(L) \rightarrow{ }^{p} \mathbf{E}^{r-1}(L)$ that satisfies

$$
H(\boldsymbol{\Delta} \omega)+\boldsymbol{\Delta} H(\omega)=\omega, \quad \omega \in{ }^{p} \mathbf{E}^{r}(L), \quad r=1, \ldots, p .
$$

All 0 -forms are mapped to zero by $H$. To begin with, consider the simplest case, in which the lattice $L$ is one-dimensional and connected (so that it is projectable); set $p=r=1$ and $\omega=f\left(n^{1}\right) \Delta_{1}$. Clearly $\omega$ is closed for arbitrary $f\left(n^{1}\right)$, so we must find a homotopy operator $H:{ }^{1} \mathbf{E}^{1}(L) \rightarrow{ }^{1} \mathbf{E}^{0}(L)$ such that

$$
\Delta(H(\omega))=\omega,
$$

for all functions $f\left(n^{1}\right)$. Let $H(\omega)=g\left(n^{1}\right)$; then (9) amounts to

$$
(S-\text { id }) g\left(n^{1}\right)=f\left(n^{1}\right),
$$

whose solution is $g\left(n^{1}\right)=g\left(n_{0}^{1}\right)+h_{1}(\omega)$, where

$$
h_{1}\left(f\left(n^{1}\right) \Delta_{1}\right)=\left\{\begin{aligned}
\sum_{k=n_{0}^{1}}^{n^{1}-1} f(k) & : n^{1}>n_{0}^{1}, \\
0 & : n^{1}=n_{0}^{1}, \\
-\sum_{k=n^{1}}^{n_{0}^{1}-1} f(k) & : n^{1}<n_{0}^{1} .
\end{aligned}\right.
$$


and $n_{0}^{1} \in L$ is an arbitrary fixed point. The arbitrary constant $g\left(n_{0}^{1}\right)$ is annihilated by $\boldsymbol{\Delta}$, so we discard it. Hence a suitable homotopy operator for $p=1$ is $H=h_{1}$. Now consider $\omega \in{ }^{1} \mathbf{E x}^{0}(L)$, so that $\omega=f\left(n^{1}\right)$ for some function $f$. Then

$$
\begin{aligned}
H \boldsymbol{\Delta}(\omega)+\Delta H(\omega) & =h_{1}\left(\left\{f\left(n^{1}+1\right)-f\left(n^{1}\right)\right\} \Delta_{1}\right) \\
& =f\left(n^{1}\right)-f\left(n_{0}^{1}\right) \\
& =\omega-\left.\omega\right|_{n^{1}=n_{0}^{1}} .
\end{aligned}
$$

In particular, if $\omega \in \operatorname{ker} \boldsymbol{\Delta}$ then the left-hand side of (10) vanishes, so

$$
\omega=\left.\omega\right|_{n^{1}=n_{0}^{1}} .
$$

Consequently Lemma 29 holds for $p=1$. We now develop homotopy operators for higher-dimensional lattices, by combining operators similar to $h_{1}$ with projection operators and using induction on $p$. Let $L$ be a $p$-dimensional projectable lattice. For simplicity, we shall assume that there is a $(p, p-1, \ldots, 1)$-ordered path from every point $\mathbf{n}=\left(n^{1}, \ldots, n^{p}\right) \in L$ to a particular point $\mathbf{n}_{0}=\left(n_{0}^{1}, \ldots, n_{0}^{p}\right) \in L$. Hence, for each $\mathbf{n}$, the lattice $L$ contains the points

$$
\begin{aligned}
\left(n^{1}, n^{2}, \ldots, n^{p-1}, k\right), & \text { for all } k \in \mathbb{Z} \text { between } n_{0}^{p} \text { and } n^{p}, \\
\left(n^{1}, n^{2}, \ldots, n^{p-2}, k, n_{0}^{p}\right), & \text { for all } k \in \mathbb{Z} \text { between } n_{0}^{p-1} \text { and } n^{p-1}, \\
\vdots & \vdots \\
\left(k, n_{0}^{2}, \ldots, n_{0}^{p-1}, n_{0}^{p}\right), & \text { for all } k \in \mathbb{Z} \text { between } n_{0}^{1} \text { and } n^{1} .
\end{aligned}
$$

It is helpful to use a formal analogue of the interior product of a vector field and a differential form, by defining operators $\left.\partial_{n^{i}}\right\lrcorner:{ }^{j} \mathbf{E x}^{r}(L) \rightarrow{ }^{j} \mathbf{E x}^{r-1}(L)$ by the relations $\left.\partial_{n^{i}}\right\lrcorner \Delta_{k}=\delta_{k}^{i}$, where $\delta$ is the Kronecker symbol. This is extended to all difference forms by linearity and the product rule. Let $h_{i}:{ }^{j} \mathbf{E x}^{r}(L) \rightarrow{ }^{j} \mathbf{E x}^{r-1}(L)$ be defined by

$$
h_{i}(\omega)=\left\{\begin{aligned}
\left.\sum_{k=n_{0}^{i}}^{n^{i}-1}\left(\partial_{n^{i}}\right\lrcorner \omega\right)\left.\right|_{n^{i}=k} & : n^{i}>n_{0}^{i}, \\
0 & : n^{i}=n_{0}^{i}, \\
\left.-\sum_{k=n^{i}}^{n_{0}^{i}-1}\left(\partial_{n^{i}}\right\lrcorner \omega\right)\left.\right|_{n^{i}=k} & : n^{i}<n_{0}^{i} .
\end{aligned}\right.
$$

Roughly speaking, $h_{i}(\omega)$ sums the $r$-form $\omega$ along the 1-chain obtained by varying the $i^{\text {th }}$ coordinate from $n_{0}^{i}$ to $n^{i}$, while leaving the other coordinates unchanged. Therefore we call the operators $h_{i}$ summation operators. Define the projection maps

$$
\Pi_{j}:{ }^{j} \mathbf{E x}^{r}(L) \rightarrow{ }^{j-1} \mathbf{E x}^{r}(L), \quad \Pi_{j}(\omega)=\left.\omega\right|_{n^{j}=n_{0}^{j}, \Delta_{j}=0}
$$

and note that

$$
\Pi_{r} \circ \Pi_{r+1} \circ \cdots \circ \Pi_{p} \omega=0
$$


for all $\omega \in{ }^{p} \mathbf{E x}^{r}(L), r \geq 1$. Our assumption that $(p, p-1, \ldots, 1)$-ordered paths exist enables us to project first in the $n^{p}$-direction, then in the $n^{p-1}$-direction and so on, down to $n^{1}$ last. This allows us to construct the homotopy map by induction on the number of edges needed to get to $\mathbf{n}$ (that is, on the dimension of the lattice). There is no essential loss of generality in doing this, for the resulting homotopy operator can be adapted to other projectable lattices merely by using the projection maps and summation operators in the required order.

Theorem 30 Under the above assumptions on the domain of definition $L$ of $\omega \in$ ${ }^{p} \mathbf{E x}^{r}(L)$, whose coefficients are functions in $\mathcal{B}$, let

$$
h(\omega)=h_{p}(\omega)+\sum_{i=1}^{p-1} h_{i}\left(\Pi_{i+1} \circ \Pi_{i+2} \circ \cdots \circ \Pi_{p} \omega\right) .
$$

Then

$$
H_{\mathcal{B}}(\omega)=\left\{\begin{aligned}
h(\omega) & \omega \in{ }^{p} \mathbf{E x}^{r}(L), r>0 \\
\left.\omega\right|_{\mathbf{n}=\mathbf{n}_{0}} & \omega \in{ }^{p} \mathbf{E x}^{0}(L)
\end{aligned}\right.
$$

is a homotopy operator for the complex ${ }^{p} \operatorname{Ex}(L)$ over $\mathcal{B}$.

Example 31 If $p=2$ then for 1-forms $\omega=\alpha\left(n^{1}, n^{2}\right) \Delta_{1}+\beta\left(n^{1}, n^{2}\right) \Delta_{2}$ the homotopy map is

$$
h(\omega)=h_{2}(\omega)+h_{1}\left(\Pi_{2}(\omega)\right)=h_{2}(\omega)+h_{1}\left(\alpha\left(n^{1}, n_{0}^{2}\right) \Delta_{1}\right)
$$

whereas for 2-forms $\omega=f\left(n^{1}, n^{2}\right) \Delta_{1} \wedge \Delta_{2}$ the homotopy map is

$$
h(\omega)=h_{2}(\omega)+h_{1}\left(\Pi_{2}(\omega)\right)=h_{2}(\omega) .
$$

\section{Notes:}

1. If $r \geq 1$ then, from (13), the sum in (14) need only be taken from $i=r$ to $i=p-1$.

2. If $\tilde{\omega}=\Pi_{p} \omega$ then

$$
h(\omega)=h_{p}(\omega)+h(\tilde{\omega})
$$

Proof: It is sufficient to prove that

$$
h(\Delta \omega)+\Delta h(\omega)=\omega-\Pi_{1} \circ \cdots \circ \Pi_{p} \omega .
$$

To see this, note that if $\omega \in{ }^{p} \mathbf{E x}^{r}(L)$ and $r \geq 1$ then, by (13), $\Pi_{1} \circ \cdots \circ \Pi_{p} \omega=0$ and thus $H_{\mathcal{B}}=h$ is a homotopy map. To show exactness at ${ }^{p} \mathbf{E x}^{0}(L)$, we need to show that

$$
h(\Delta \omega)+\left.\omega\right|_{\mathbf{n}=\mathbf{n}_{0}}=\omega
$$

for $\omega \in{ }^{p} \mathbf{E x}^{0}(L)$. But this is precisely (17), because

$$
\omega \in{ }^{p} \mathbf{E x}^{0}(L) \Longrightarrow h(\omega)=0, \quad \Pi_{1} \circ \cdots \circ \Pi_{p} \omega=\left.\omega\right|_{\mathbf{n}=\mathbf{n}_{0}} .
$$


The proof of (17) is by induction on $p$. First note that if $\omega \in{ }^{1} \mathbf{E x}^{0}(L)$ then $\omega=f\left(n^{1}\right)$ for some function $f$, and therefore

$$
\begin{aligned}
h(\Delta \omega)+\Delta h(\omega) & =h\left(\left\{f\left(n^{1}+1\right)-f\left(n^{1}\right)\right\} \Delta_{1}\right) \\
& =f\left(n^{1}\right)-f\left(n_{0}^{1}\right) \\
& =\omega-\left.\omega\right|_{n^{1}=n_{0}^{1}} \\
& =\omega-\Pi_{1} \omega .
\end{aligned}
$$

Also if $\omega \in{ }^{p} \mathbf{E x}^{p}(L)$ then $\omega$ is a multiple of the $p$-form $\Delta_{1} \wedge \Delta_{2} \wedge \cdots \wedge \Delta_{p}$ and so $\Pi_{p} \omega=0$ and $\boldsymbol{\Delta} \omega=0$. Hence

$$
\begin{aligned}
h(\Delta \omega)+\Delta h(\omega) & =\Delta h_{p}(\omega) \\
& =\omega \\
& =\omega-\Pi_{1} \circ \cdots \circ \Pi_{p} \omega .
\end{aligned}
$$

Now fix $r<p$ and suppose that $H_{\mathcal{B}}$ is a homotopy operator for all $p^{\prime}<p$. We set $\tilde{\omega}=\Pi_{p}(\omega)$ and observe that $\tilde{\omega} \in{ }^{p-1} \mathbf{E x}^{r}(L)$; the induction hypothesis implies that

$$
h(\boldsymbol{\Delta} \tilde{\omega})+\boldsymbol{\Delta} h(\tilde{\omega})=\tilde{\omega}-\Pi_{1} \circ \cdots \circ \Pi_{p-1} \tilde{\omega}
$$

The last term is nonzero only if $r=0$. Note that

$$
\begin{aligned}
\Pi_{p}(\Delta \omega) & =\Pi_{p}\left(\sum_{j=1}^{p} \Delta_{j} \wedge\left(S_{j}-\mathrm{id}\right) \omega\right) \\
& =\sum_{j=1}^{p-1} \Delta_{j} \wedge\left(S_{j}-\mathrm{id}\right)\left(\Pi_{p} \omega\right) \\
& =\boldsymbol{\Delta} \tilde{\omega}
\end{aligned}
$$

and so, from (16),

$$
\begin{aligned}
h(\boldsymbol{\Delta} \omega) & =h_{p}(\boldsymbol{\Delta} \omega)+h\left(\Pi_{p}(\boldsymbol{\Delta} \omega)\right) \\
& =h_{p}(\boldsymbol{\Delta} \omega)+h(\boldsymbol{\Delta} \tilde{\omega}) .
\end{aligned}
$$

Also from (16),

$$
\Delta h(\omega)=\Delta h_{p}(\omega)+\Delta h(\tilde{\omega}),
$$

and therefore, using (20),

$$
\begin{aligned}
h(\boldsymbol{\Delta} \omega)+\boldsymbol{\Delta} h(\omega) & =h_{p}(\boldsymbol{\Delta} \omega)+\boldsymbol{\Delta} h_{p}(\omega)+\tilde{\omega}-\Pi_{1} \circ \Pi_{2} \circ \cdots \circ \Pi_{p-1} \tilde{\omega} \\
& =h_{p}(\boldsymbol{\Delta} \omega)+\Delta h_{p}(\omega)+\Pi_{p}(\omega)-\Pi_{1} \circ \Pi_{2} \circ \cdots \circ \Pi_{p} \omega .
\end{aligned}
$$

So to prove the correctness of the homotopy formula, we need only show that

$$
h_{p}(\boldsymbol{\Delta} \omega)+\boldsymbol{\Delta} h_{p}(\omega)=\omega-\Pi_{p} \omega .
$$


This can be verified by direct calculation, as follows.

$$
\begin{aligned}
h_{p}(\boldsymbol{\Delta} \omega) & +\boldsymbol{\Delta} h_{p}(\omega) \\
& \left.\left.\left.=\partial_{n^{p}}\right\lrcorner\left(\Delta_{p} \wedge \omega\right)-\left(\partial_{n^{p}}\right\lrcorner\left(\Delta_{p} \wedge \omega\right)\right)\left.\right|_{n^{p}=n_{0}^{p}}+\Delta_{p} \wedge\left(\partial_{n^{p}}\right\lrcorner \omega\right) \\
& =\omega-\left.\omega\right|_{n^{p}=n_{0}^{p}, \Delta_{p}=0} \\
& =\omega-\Pi_{p} \omega
\end{aligned}
$$

as required, where we have used the identity

$$
\left.\left.\partial_{n^{p}}\right\lrcorner\left(\Delta_{j} \wedge \eta\right)+\Delta_{j} \wedge\left(\partial_{n^{p}}\right\lrcorner \eta\right)=\delta_{j}^{p} \eta .
$$

Equations (18) and (19) show that (17) holds for $p=r$ if $r \geq 1$ and for $p=1$ if $r=0$. By induction, (17) holds for all $p, r$, as required.

\section{From local to global}

As for de Rham cohomology of a manifold, the difference cohomology of a lattice variety may be calculated in terms of the "pattern of intersections" of a "good cover", a notion formalised in the definition of the Cech cohomology of the cover. We follow the line of argument in Weil's celebrated proof [36], as expounded in [5], pointing out the relevant technical differences.

As a corollary, we show that if the cover of a space and its lattice approximation has the same Cech cohomology, then the $\boldsymbol{\Delta}$-cohomology will match the smooth de Rham cohomology in a well-defined way. There are, however, limits to the analogy with the smooth case, which the examples show. In the next section, we calculate examples using the techniques developed in this section.

\subsection{The Čech difference operator}

The purpose of the Cech difference operator is to measure when forms defined locally can be "glued" or pieced together to make forms with extended domains.

Let $L_{\alpha}, \alpha \in \mathcal{A}$, be a finite collection of lattices composed of sums of cells. In our application, these will be the pieces that comprise a lattice variety. Denote

$$
\begin{array}{ccl}
L_{\alpha_{0}} \cap L_{\alpha_{1}} & \text { by } & L_{\alpha_{0} \alpha_{1}} \\
L_{\alpha_{0}} \cap L_{\alpha_{1}} \cap L_{\alpha_{2}} & \text { by } & L_{\alpha_{0} \alpha_{1} \alpha_{2}}
\end{array}
$$

and so forth, and let $\amalg$ denote disjoint union. 
The inclusions $\imath_{i}: L_{\alpha_{0} \cdots \alpha_{i} \cdots \alpha_{j}} \rightarrow L_{\alpha_{0} \cdots \widehat{\alpha_{i}} \cdots \alpha_{j}}$ give rise to the sequence

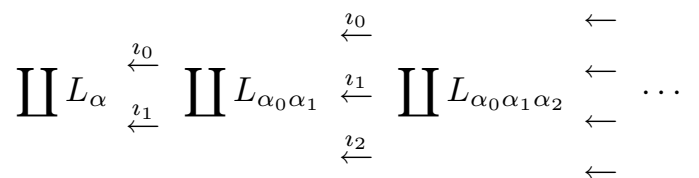

These in turn give rise to a sequence of restrictions of difference forms

$$
\bigoplus \mathbf{E x}^{*}\left(L_{\alpha}\right) \underset{\stackrel{\delta_{1}}{\rightarrow}}{\stackrel{\delta_{0}}{\rightarrow}} \bigoplus \mathbf{E x}^{*}\left(L_{\alpha_{0} \alpha_{1}}\right) \stackrel{\stackrel{\delta_{0}}{\rightarrow}}{\stackrel{\delta_{1}}{\rightarrow}} \ldots
$$

where, for example,

$$
\delta_{0}: \mathbf{E x}^{*}\left(L_{\alpha_{1} \alpha_{2}}\right) \rightarrow \mathbf{E x}^{*}\left(L_{\alpha_{0} \alpha_{1} \alpha_{2}}\right)
$$

Definition 32 The Čech difference operator

$$
\delta: \bigoplus \mathbf{E x}^{*}\left(L_{\alpha_{0} \cdots \alpha_{p}}\right) \rightarrow \bigoplus \mathbf{E x}^{*}\left(L_{\alpha_{0} \cdots \alpha_{p+1}}\right)
$$

is defined for each integer $p \geq 0$. If $\omega \in \bigoplus \mathbf{E x}^{*}\left(L_{\alpha_{0} \cdots \alpha_{p}}\right)$ has components

$$
\omega_{\alpha_{0} \cdots \alpha_{p}} \in \mathbf{E x}^{q}\left(L_{\alpha_{0} \cdots \alpha_{p}}\right)
$$

then $\delta \omega$ has components

$$
(\delta \omega)_{\alpha_{0} \cdots \alpha_{p+1}}=\left.\sum_{i=0}^{p+1}(-1)^{i} \omega_{\alpha_{0} \cdots \widetilde{\alpha_{i}} \cdots \alpha_{p+1}}\right|_{L_{\alpha_{0} \cdots \alpha_{p+1}}}
$$

Important Remark 33 The key difference to the smooth case is that here, the restriction sets to zero any inappropriate forms at points which are TopRight points in the range space.

Theorem 34 The Čech difference operator $\delta$ satisfies $\delta^{2}=0$

Example 35 For the lattice covers shown, we give the Čech difference operator. 
(i)

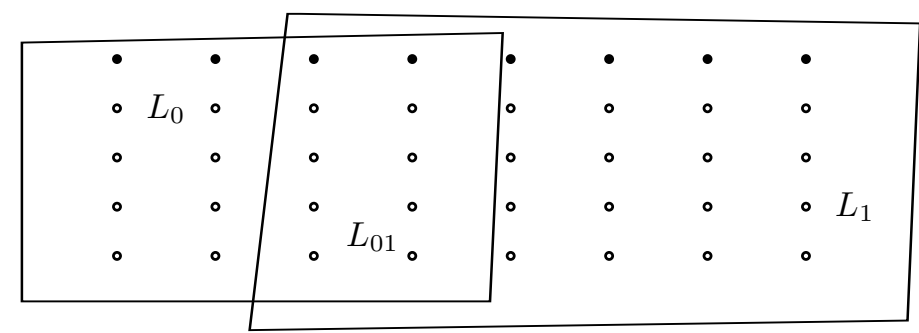

$$
\omega=\left(\omega_{0}, \omega_{1}\right), \quad(\delta \omega)_{01}=\left.\left(\omega_{1}-\omega_{0}\right)\right|_{L_{01}}
$$

(ii)

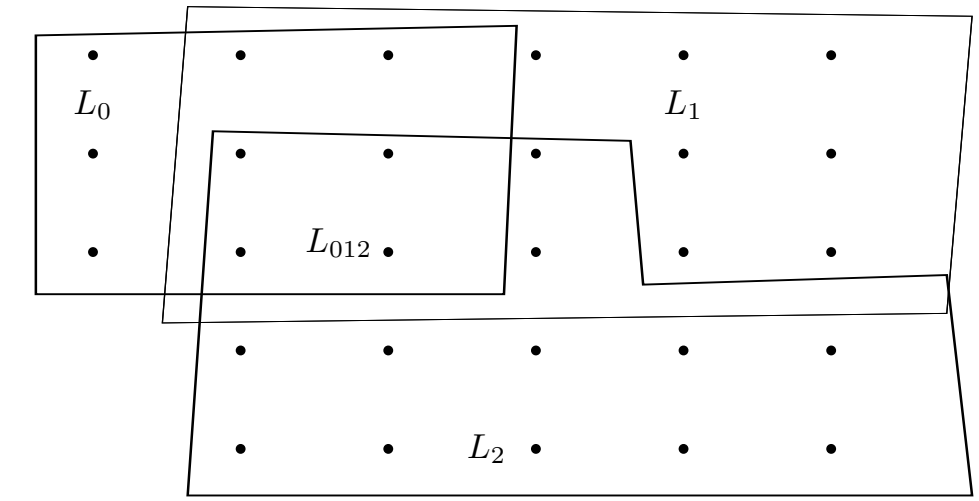

$$
\omega=\left(\omega_{01}, \omega_{12}, \omega_{02}\right), \quad(\delta \omega)_{012}=\left.\left(\omega_{12}-\omega_{02}+\omega_{01}\right)\right|_{L_{012}}
$$

Theorem 36 The sequence

$$
\bigoplus \mathbf{E x}^{i}\left(L_{\alpha}\right) \stackrel{\delta}{\longrightarrow} \bigoplus \mathbf{E x}^{i}\left(L_{\alpha \beta}\right) \stackrel{\delta}{\longrightarrow} \bigoplus \mathbf{E x}^{i}\left(L_{\alpha \beta \gamma}\right) \cdots
$$

is exact for each $i$.

The proof of exactness of the Čech sequence requires a partition of unity, $\left\{\rho_{\alpha}\right\}$,

$$
\sum \rho_{\alpha}=1, \quad \operatorname{supp}\left(\rho_{\alpha}\right) \subseteq L_{\alpha}
$$

For $\omega \in \bigoplus \mathbf{E x}^{i}\left(L_{\alpha_{0} \cdots \alpha_{p}}\right)$, define

$$
(K \omega)_{\alpha_{0} \cdots \alpha_{p-1}}=\sum_{\alpha} \rho_{\alpha} \omega_{\alpha \alpha_{0} \cdots \alpha_{p-1}}
$$

Then

$$
\delta K+K \delta=\mathrm{id}
$$

except perhaps at TopRight points in the range spaces where the restriction maps send some forms to zero. To ensure $(22)$ is valid for all points, so that $K$ is a homotopy 
operator for the sequence (21), the functions $\rho_{\alpha}$ must take the value 1 at any point $P$ such that

$$
\left.\omega\right|_{L_{\alpha}}(P) \neq\left.\omega\right|_{L_{\alpha \beta}}(P) .
$$

Such a point is shown in the next Figure, where $P$ is a TopRight point in $L_{\alpha}$ but not $L_{\alpha} \cup L_{\beta}$.

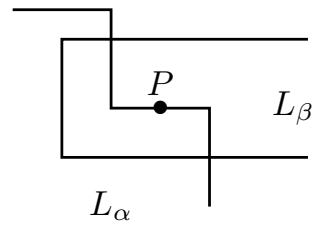

For example, if $\omega(P)=a \Delta_{1}+b \Delta_{2}$ in $L_{\beta}$, then $\left.\omega\right|_{L_{\alpha \beta}}=a \Delta_{1}$, because $\Delta_{2}$ is not defined at $P$ in $L_{\alpha \beta}$. This constraint on the $\rho$ poses no difficulties as unlike the smooth case, the partition functions need not be continuous.

Example 37 Example 11(ii) cont. Suppose

$$
\delta\left(\omega_{12}, \omega_{02}, \omega_{01}\right)=\left.\left(\omega_{12}-\omega_{02}+\omega_{01}\right)\right|_{L_{012}}=0,
$$

then there exist $\omega_{0} \in \mathbf{E x}^{i}\left(L_{0}\right), \omega_{1} \in \mathbf{E x}^{i}\left(L_{1}\right)$ and $\omega_{2} \in \mathbf{E x}^{i}\left(L_{2}\right)$ such that

$$
\begin{aligned}
& \omega_{12}=\omega_{1}-\omega_{2} \\
& \omega_{01}=\omega_{1}-\omega_{0} \\
& \omega_{02}=\omega_{0}-\omega_{2}
\end{aligned}
$$

Theorem 38 The Čech difference operator $\delta$ and the difference operator $\boldsymbol{\Delta}$ commute, that is,

$$
\Delta \delta=\delta \Delta
$$

This follows as the smooth case, except at TopRight points, where the restrictions ensure commutativity.

\section{2 Čech cochains}

For a lattice variety $L$ with cover $\left\{L_{\alpha} \mid \alpha \in A\right\}$, where $A$ is the index set for the cover, let $\mathcal{L}$ denote the set $\left\{L_{\alpha_{0} \alpha_{1} \cdots \alpha_{r}} \mid r \in \mathbb{N}, \alpha_{i} \in A\right\}$. Further, let $\mathcal{L}^{i}=\left\{L_{\alpha_{0} \alpha_{1} \cdots \alpha_{i}}\right\}$.

For a set $S$, let

$$
\langle S\rangle_{\mathbb{R}}=\left\{\sum a_{s} s \mid s \in S, a_{s} \in \mathbb{R}\right\}
$$

be the set of formal sums of the elements of $S$ with coefficients in $\mathbb{R}$. 
Definition 39 Čech cochains are defined as

$$
\check{\mathcal{C}}^{i}(\mathcal{L})=\left\{f:\left\langle\mathcal{L}^{i}\right\rangle \rightarrow \mathbb{R}\right\}, \quad i \in \mathbb{N}
$$

The Čech $p$-cochains can be viewed as constant valued degree zero $\Delta$-forms on the $p+1$ intersections. Let $s$ denote the injection map,

$$
s: \check{\mathcal{C}}^{i}(\mathcal{L}) \rightarrow \oplus \mathbf{E x}^{0}\left(L_{\alpha_{0} \alpha_{1} \cdots \alpha_{i}}\right) .
$$

The maps $\delta: \check{\mathcal{C}}^{i} \rightarrow \check{\mathcal{C}}^{i+1}$ are then restrictions of the Čech difference operator given in Definition 32 above to the $\boldsymbol{\Delta}$ forms with which they are identified via the map $s$, so that $s \delta=\delta s$. Note also that $\boldsymbol{\Delta} \circ s=0$, because the image of $s$ is locally constant.

Definition 40 The Čech cohomology groups of $\mathcal{L}$ are defined to be

$$
\check{H}^{i}(\mathcal{L})=\frac{\left.\operatorname{ker} \delta\right|_{\check{\mathcal{C}}^{i}(\mathcal{L})}}{\left.\operatorname{im} \delta\right|_{\check{\mathcal{C}}^{i-1}(\mathcal{L})}}
$$

Given a particular cover of $L$, once the $L_{\alpha}$ and their intersections are known, the Cech cohomology of $\mathcal{L}$ is easily worked out. This first example is a simple expository one for the sake of completeness.

Example 41 Consider the following diagram of lattices and their intersections,

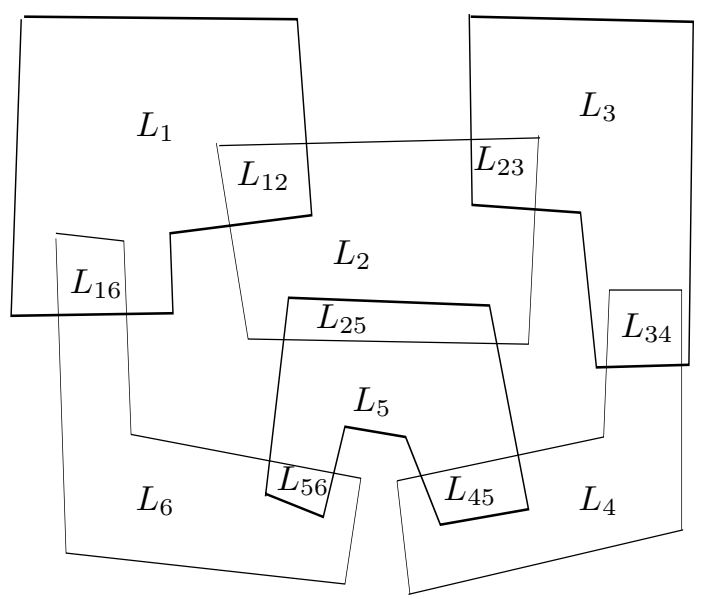

In this example, $\check{\mathcal{C}}^{0} \cong \mathbb{R}^{6}$ and $\check{\mathcal{C}}^{1} \cong \mathbb{R}^{7}$. The $\check{C}$ ech sequence is

$$
0 \longrightarrow \check{\mathcal{C}}^{0} \stackrel{\delta}{\longrightarrow} \check{\mathcal{C}}^{1} \longrightarrow 0
$$


where

$$
\begin{aligned}
& \begin{array}{llllll}
L_{1} & L_{2} & L_{3} & L_{4} & L_{5} & L_{6}
\end{array} \\
& \delta=\begin{array}{l}
L_{12} \\
L_{23} \\
L_{34} \\
L_{45} \\
L_{25} \\
L_{56} \\
L_{16}
\end{array}\left(\begin{array}{cccccc}
1 & -1 & 0 & 0 & 0 & 0 \\
0 & 1 & -1 & 0 & 0 & 0 \\
0 & 0 & 1 & -1 & 0 & 0 \\
0 & 0 & 0 & 1 & -1 & 0 \\
0 & 1 & 0 & 0 & -1 & 0 \\
0 & 0 & 0 & 0 & 1 & -1 \\
1 & 0 & 0 & 0 & 0 & -1
\end{array}\right)
\end{aligned}
$$

Let $\chi_{\alpha}$ be the characteristic function for $L_{\alpha}$, which is the function that takes the value 1 on $L_{\alpha}$ and is zero elsewhere. Then

$$
\check{H}^{0}(\mathcal{L})=\operatorname{ker} \delta=\sum_{j=1}^{6} \chi_{j}
$$

and

$$
\begin{aligned}
& \check{H}^{1}(\mathcal{L})=\check{\mathcal{C}}^{1} / \mathrm{im} \delta \\
& \cong\left\langle\chi_{12}+\chi_{25}+\chi_{56}+\chi_{16}, \chi_{12}+\chi_{23}+\chi_{34}+\chi_{45}+\chi_{56}+\chi_{16}\right\rangle_{\mathbb{R}}
\end{aligned}
$$

These may be calculated in a symbolic computing environment using commands for the kernel, transpose and column space on the matrix form of $\delta$.

In $\S 2$, we stressed the need for a valid cover of the lattice variety. Consider the variety shown in Figure 2 in $§ 2.3 .3$. As $L_{1} \cap L_{2}$ has two components, the Cech cohomology is the same as a two-dimensional annulus, which is not isomorphic to that of a corner. Thus the Cech cohomology captures the fact that there is a central point at which 2 -forms are not defined.

\subsection{The Čech- $\Delta$ double complex}

The double complex we describe in this section is the "Divide and Conquer" method for calculating cohomology groups, pioneered by Weil [36].

Let $L$ be a lattice variety with cover $\left\{L_{\alpha}\right\}$.

Definition 42 If the cover $\mathcal{L}$ of $L$ is not only valid but has the property that each $L_{\alpha_{0} \cdots \alpha_{r}}$ has trivial $\boldsymbol{\Delta}$-cohomology, we say that $\mathcal{L}$ is a good cover of $L$.

Example 43 The cover of the pillow model of the cube consisting of the front and back faces, is not a good cover. The intersection is a one dimensional annular lattice which has nontrivial $\boldsymbol{\Delta}$ cohomology.

Good covers of the punctured plane, the annular lattice and the cube model of the sphere will be given in $\S 5$.

Define $r: \mathbf{E x}^{i}(L) \rightarrow \bigoplus \mathbf{E x}^{i}\left(L_{\alpha}\right)$, by

$$
r(\omega)=\left(\left.\omega\right|_{L_{0}},\left.\omega\right|_{L_{1}}, \ldots\right) .
$$


Then $r \boldsymbol{\Delta}=\boldsymbol{\Delta} r$. Combining results from the previous discussions, we find that the following diagram is commutative, where $\boldsymbol{\Delta}$ on a direct sum is assumed to act componentwise.

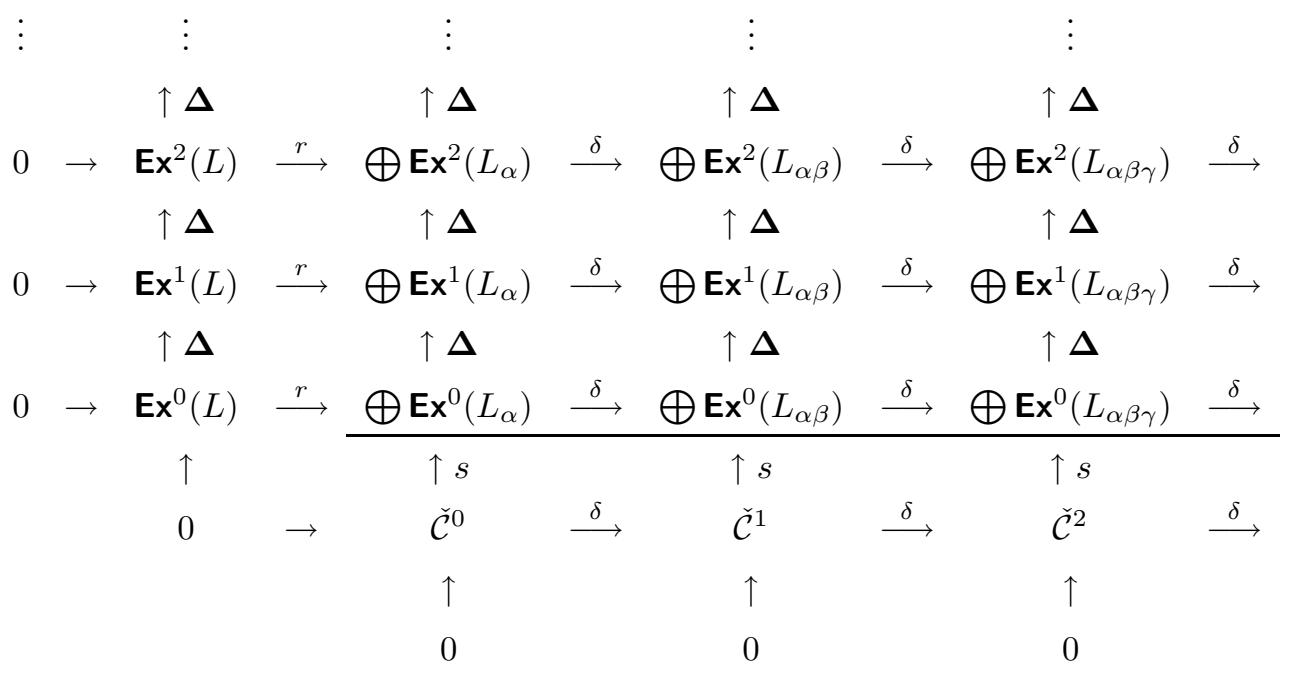

The first important point is that those parts of the columns above the long horizontal line are exact by definition of a good cover. Those parts of the rows above the line are exact as they are the Cech sequences. The second important point is that the kernels of the $\boldsymbol{\Delta}$ in the leftmost column are, by definition, the global $\boldsymbol{\Delta}$-forms.

The complex is used to construct a global $\Delta$-form from a Čech form and vice versa. As an example, consider now the descent diagram for $p=2$ beginning on the left with $\omega \in \mathbf{E x}^{2}(L):$

$$
\begin{aligned}
& 0=\boldsymbol{\Delta}(\omega) \quad 0 \\
& \uparrow \boldsymbol{\Delta} \quad \uparrow \boldsymbol{\Delta} \\
& \omega \in \operatorname{Ex}^{2}(L) \stackrel{r}{\longrightarrow}\left(\left.\omega\right|_{L_{0}}, \ldots\right) \quad \longrightarrow \quad 0 \\
& \uparrow \boldsymbol{\Delta} \\
& \left(\eta_{0}, \eta_{1}, \ldots\right) \stackrel{\stackrel{\delta}{\rightarrow}}{\left(\xi_{01}, \ldots\right)} \underset{\uparrow \boldsymbol{\Delta}}{\stackrel{\delta}{\rightarrow}} \quad \text { } \\
& \frac{\left(\chi_{01}, \ldots\right) \stackrel{\delta}{\rightarrow}\left(\phi_{012}, \ldots\right) \stackrel{\delta}{\rightarrow} 0}{\uparrow s} \\
& \phi \in \check{\mathcal{C}}^{2} \quad \stackrel{\delta}{\rightarrow} 0
\end{aligned}
$$

Assume that $\Delta \omega=0$, that is, $\omega$ is a closed $\Delta$-form. As each $\left.\omega\right|_{L_{\alpha}}$ is closed on $L_{\alpha}$ which has trivial $\Delta$-cohomology, we use the relevant homotopy operator to obtain $\eta_{\alpha}$ 
on $L_{\alpha}$ such that $\boldsymbol{\Delta}\left(\eta_{\alpha}\right)=\left.\omega\right|_{L_{\alpha}}$. Applying $\delta$ to $\left(\eta_{0}, \eta_{1}, \ldots\right)$ yields $\xi=\left(\xi_{01}, \ldots\right)$, say. By commutativity of the diagram, each component of $\xi$ is closed on its domain. Thus, there exists for each $\xi_{\alpha \beta}$, a $\chi_{\alpha \beta}$, such that on $L_{\alpha \beta}, \boldsymbol{\Delta}\left(\chi_{\alpha \beta}\right)=\xi_{\alpha \beta}$. Taking $\delta\left(\chi_{01}, \ldots\right)$ yields

$$
\phi=\left(\phi_{012}, \ldots\right) \in \bigoplus \operatorname{Ex}^{0}\left(L_{\alpha_{0} \alpha_{1} \alpha_{2}}\right)
$$

such that $\boldsymbol{\Delta}(\phi)=0$ and $\delta(\phi)=0$.

As zero-forms in ker $\boldsymbol{\Delta}$ are just constants, it follows that $\phi$ can be identified with a Čech cocycle,

$$
\phi:\left\langle\left\{L_{\alpha \beta \gamma}\right\}\right\rangle_{\mathbb{R}} \rightarrow \mathbb{R}, \quad \phi\left(L_{\alpha \beta \gamma}\right)=\phi_{\alpha \beta \gamma} .
$$

Standard algebraic arguments are used to prove the following theorem.

Theorem $44 A \boldsymbol{\Delta}$ p-cocycle produces a Čech p-cocycle via the descent diagram. Moreover, exact $\boldsymbol{\Delta}$ cocycles are mapped to exact Čech cocycles.

As the Cech cohomology is easy to compute, we would like to use it to compute the $\Delta$-cohomology of the lattice variety. To this end, we reverse the descent procedure to obtain an ascent procedure. Instead of using the homotopy maps for exact $\boldsymbol{\Delta}$ sequences (the columns), we now use those for the exact Cech sequences (the rows). Then a standard argument yields the following theorem.

Theorem 45 If $\mathcal{L}$ is a good cover for the lattice variety $L$, then

$$
H_{\boldsymbol{\Delta}}^{p}(L) \cong \check{H}^{p}(\mathcal{L}) .
$$

\section{$5 \quad$ Examples}

We now look at three examples to demonstrate the process of finding the $\boldsymbol{\Delta}$-cohomology from the Čech cohomology.

\subsection{The punctured plane}

Consider the cover of the punctured plane given below. 


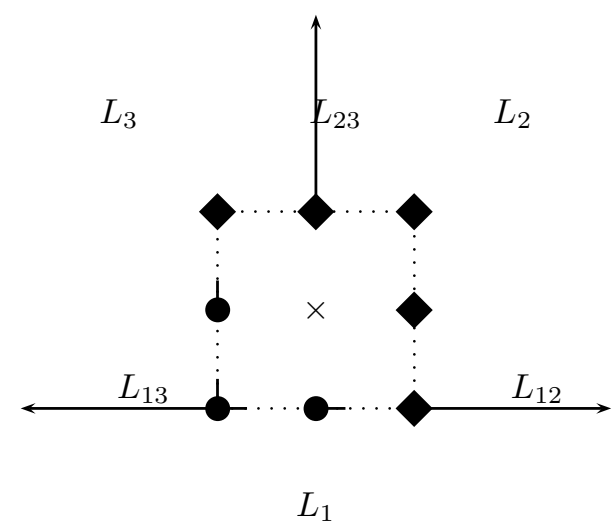

The diagram shows a good cover $\mathcal{L}$ for the "punctured plane" $L=\mathbb{Z}^{2} \backslash\{(0,0)\}$. The origin (marked by a cross) is removed, and we have shown only the points that surround it (at all other points, 2 -forms can be defined). The double intersections are along the lines, as marked, and there are no triple intersections. It should be noted that no 2 -form at $(0,-1)$ can be defined. As in $\S 2$, points where 2 -forms are definable are marked by diamonds, points where only 1 -forms are definable are marked by discs.

The Čech cohomology of $\mathcal{L}$ is

$$
\begin{aligned}
& \check{H}^{i}(\mathcal{L})=0, \quad i \geq 2 \\
& \check{H}^{1}(\mathcal{L}) \cong\langle\eta\rangle_{\mathbb{R}} \\
& \check{H}^{0}(\mathcal{L}) \cong \mathbb{R} \quad(\text { constant 0-forms) }
\end{aligned}
$$

where $\left.\eta\right|_{L_{13}}=1$ and $\left.\eta\right|_{L_{23}}=\left.\eta\right|_{L_{12}}=0$.

The ascent diagram is

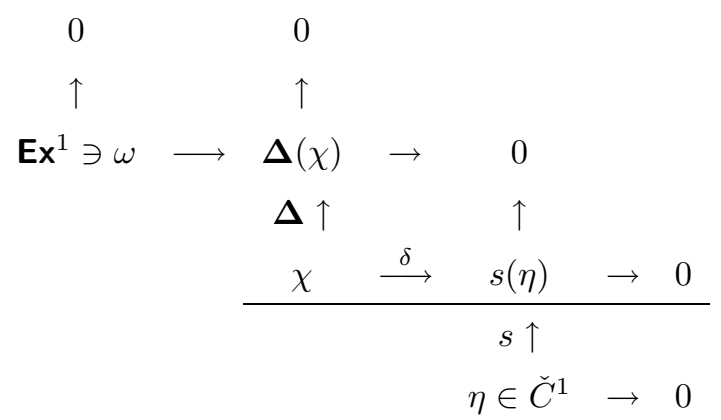


The first step is to find a pre-image $\chi$, under $\delta$, of $s(\eta)$, which is the constant 0 -form $(0,1,0) \in \mathbf{E x}^{0}\left(L_{12}\right) \oplus \mathbf{E x}^{0}\left(L_{13}\right) \oplus \mathbf{E x}^{0}\left(L_{23}\right)$. We may take

$$
\chi=\left(\chi_{1}, \chi_{2}, \chi_{3}\right) \in \mathbf{E x}^{0}\left(L_{1}\right) \oplus \mathbf{E x}^{0}\left(L_{2}\right) \oplus \mathbf{E x}^{0}\left(L_{3}\right),
$$

to be

$$
\chi_{1} \equiv 0, \quad \chi_{2} \equiv 0
$$

and

$$
\chi_{3}\left(n_{1}, n_{2}\right)= \begin{cases}0 & \left(n_{1}, n_{2}\right) \in L_{3} \backslash L_{13} \\ 1 & \left(n_{1}, n_{2}\right) \in L_{13}\end{cases}
$$

The next step is to calculate $\boldsymbol{\Delta} \chi$, which is done component-wise. We have $\boldsymbol{\Delta} \chi_{1}=$ $\Delta \chi_{2} \equiv 0$, while

$$
\Delta \chi_{3}\left(n_{1}, n_{2}\right)=\left\{\begin{aligned}
0 & & \left(n_{1}, n_{2}\right) & \in L_{1} \backslash L_{13} \\
-\Delta_{2} & & \left(n_{1}, n_{2}\right) & \in L_{13}
\end{aligned}\right.
$$

We now observe that $\boldsymbol{\Delta} \chi$ is the component wise restriction of a global closed 2-form $\omega$, given by

$$
\omega\left(n_{1}, n_{2}\right)=\left\{\begin{array}{cl}
0 & \left(n_{1}, n_{2}\right) \in L \backslash L_{13} \\
-\Delta_{2} & \left(n_{1}, n_{2}\right) \in L_{13}
\end{array}\right.
$$

To see this, first note that $\left.\omega\right|_{L_{1}} \equiv 0$, because 1-forms on the top boundary of $L_{1}$ may involve $\Delta_{1}$ only, the restriction sets any $\Delta_{2}$ terms to zero. Secondly, $\Delta \omega \equiv 0$ because 2 -forms are not defined at $(0,-1)$.

We remark that taking $\chi_{3}$, which is defined on $L_{3}$, to be

$$
\chi_{3}\left(n_{1}, n_{2}\right)=\left\{\begin{array}{cc}
0 & n_{1}, n_{2}>0 \\
1 & n_{2}=0,-1
\end{array}\right.
$$

yields a representative for $H_{\boldsymbol{\Delta}}^{1}$ which is more obviously both closed and the restriction of a global form, such as that shown in Example 19 in $\S 2.4$. Our choice of a good cover and pre-image highlights some of the subtleties of calculations with difference forms which are not present in the smooth case.

Different covers, different representatives $\eta$ of $\check{H}^{1}$ and different pre-images of $\eta$ all yield different representatives of $H^{1}(L)$. However, the difference of any two representatives of $H^{1}(L)$ is an exact form.

\subsection{An annular lattice}

A good cover of the annular lattice $L$ shown below consists of three lattice pieces, $L_{\alpha}$, $\alpha=1,2,3$, as in the diagram, with no triple intersections. (The edges and shading are included only to make the picture clear.) We assume that the co-ordinates on the $L_{\alpha}$ 
are standard so that 2 -forms are defined at the lower left points of every cube. The Cech cohomology of $\mathcal{L}$ is the same as that for the punctured plane, given in equation (23).

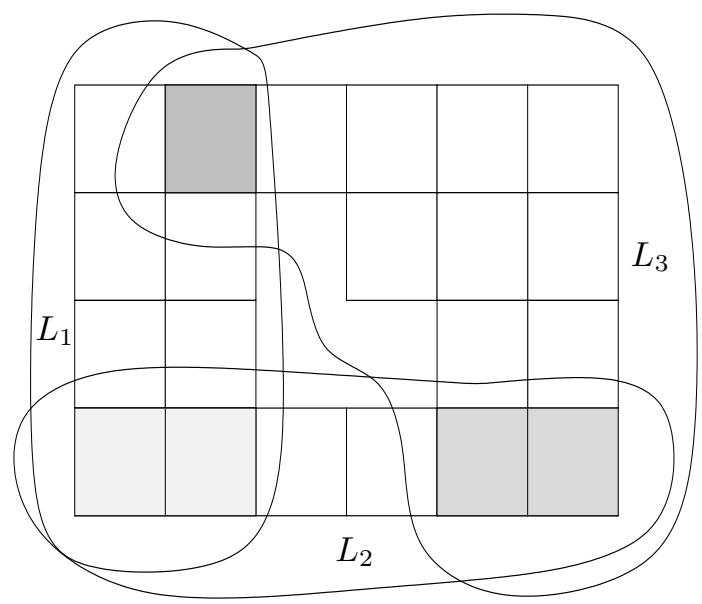

The ascent diagram is the same as given in (24) in $\S 4.1$. For variety, we take a different representative of $\check{H}^{1}$, which takes the value 1 on $L_{23}$ and is zero elsewhere.

The first step is to find a pre-image $\chi$, under $\delta$, of $s(\eta)$, which is the constant 0 -form $(0,0,1) \in \mathbf{E x}^{0}\left(L_{12}\right) \oplus \mathbf{E} \mathbf{x}^{0}\left(L_{13}\right) \oplus \mathbf{E} \mathbf{x}^{0}\left(L_{23}\right)$. We may take

$$
\chi=\left(\chi_{1}, \chi_{2}, \chi_{3}\right) \in \mathbf{E x}^{0}\left(L_{12}\right) \oplus \mathbf{E x}^{0}\left(L_{13}\right) \oplus \mathbf{E x}^{0}\left(L_{23}\right),
$$

to be

$$
\chi_{2} \equiv 1, \quad \chi_{3} \equiv 0
$$

and $\chi_{1}$, which is defined on $L_{1}$, is given diagrammatically by

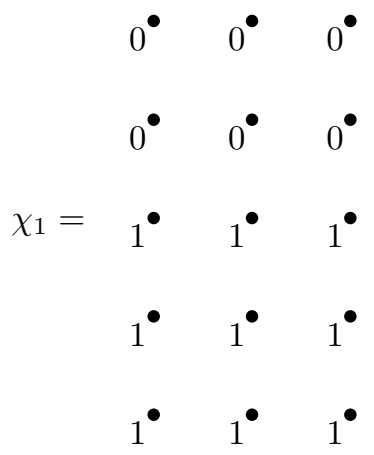

The next step is to calculate $\boldsymbol{\Delta} \chi$ component wise. This yields,

$$
\Delta \chi_{2} \equiv 0, \quad \Delta \chi_{3} \equiv 0
$$

and 


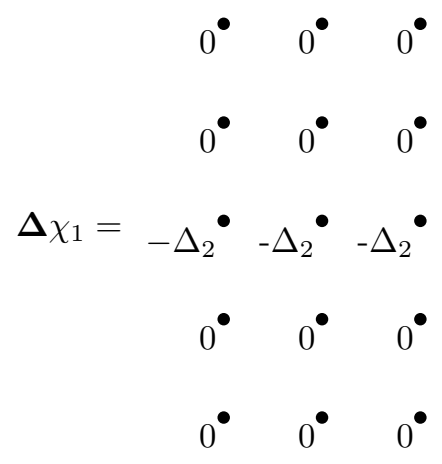

We can now see that $\boldsymbol{\Delta} \chi$ is the restriction onto components of a global form $\omega$, because on the intersections $L_{i j}$, the values of the $\boldsymbol{\Delta} \chi_{i}$ are identical. This form is closed but not exact 1-form, because we began with a closed but not exact Cech 1-form.

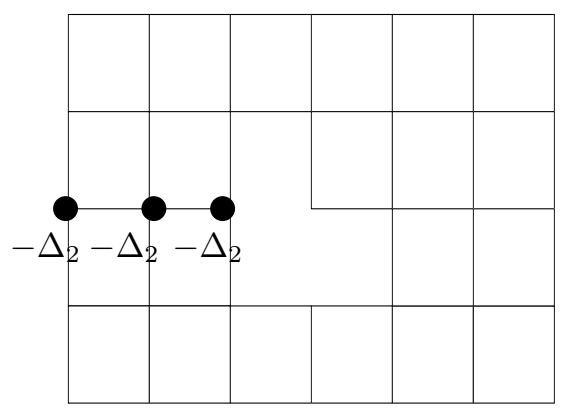

The global 2-form $\omega$; the value at unmarked points is zero.

\subsection{The lattice sphere}

The cube model of the two-dimensional lattice sphere was pictured in Example 20 (§2.3.3). Suppose the cover is given as: $L_{1}$ is the top face, $L_{5}$ is the bottom face, while $L_{2}, L_{3}$ and $L_{4}$ wrap around the "belly". The intersections $L_{i j}$ are given below, followed by a diagram of the triple intersections. This example shows another significant difference from the smooth case, which is that the intersections do not need to have the same dimension as the original variety. 

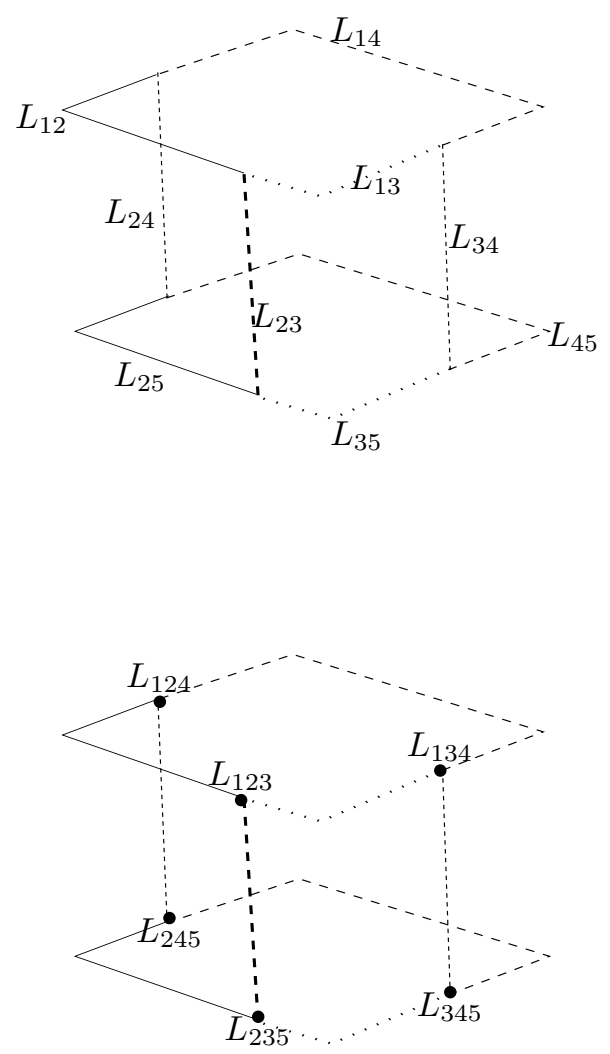

The triple intersections, labelled on the double intersection skeleton.

From the intersections of the cover, it is simple to calculate the Cech cohomology of $\mathcal{L}$,

$$
\check{H}^{i}=\left\{\begin{array}{ll}
0, & i \neq 0,2 \\
\mathbb{R} & i=0,2
\end{array} .\right.
$$

We now construct a closed but not exact difference 2 -form via the ascent procedure. The ascent diagram for $p=2$ is given in $\S 3.3$.

Step 1 A representative of $\check{H}^{2}$ is given by $\phi_{134}=1$ on $L_{134}$ and zero else.

Step 2 Construct a preimage $\chi \in \oplus \mathbf{E x}^{0}\left(L_{\alpha \beta}\right)$ under $\delta$ of $\eta$. This may be taken to be zero 
on all $L_{\alpha \beta}$ except $L_{34}$, where it is as follows:

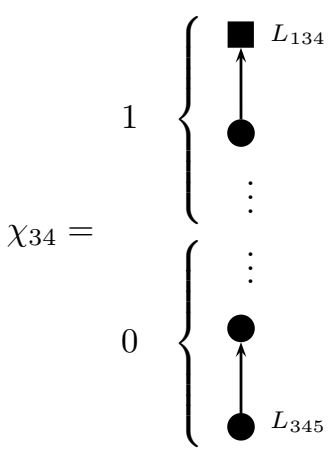

Indeed,

$$
\begin{aligned}
(\delta \chi)_{134} & =\left.\left(\chi_{34}-\chi_{14}-\chi_{13}\right)\right|_{134} \\
& =1 \\
(\delta \chi)_{235} & =\left.\left(\chi_{35}-\chi_{25}-\chi_{23}\right)\right|_{235} \\
& =0
\end{aligned}
$$

and so forth.

Step 3 Calculate the components of $\xi=\Delta \chi$ :

$$
\begin{aligned}
& 0 \quad\left\{\begin{array}{l}
\bullet\left(L_{134}\right) \\
\vdots
\end{array}\right. \\
& \boldsymbol{\Delta}_{34}=\Delta_{2} \\
& 0 \quad\left\{\begin{array}{l}
\bullet \\
\bullet\left(L_{345}\right)
\end{array}\right.
\end{aligned}
$$

and zero elsewhere.

Step 4 Calculate the components of a preimage, $\eta$, under $\delta$ of $\boldsymbol{\Delta} \chi$ in $\oplus \mathbf{E x}^{1}\left(L_{i}\right)$. This can be taken to be zero on all $L_{i}$ for $i \neq 4$, and on $L_{4}$ is given in the figure following; 


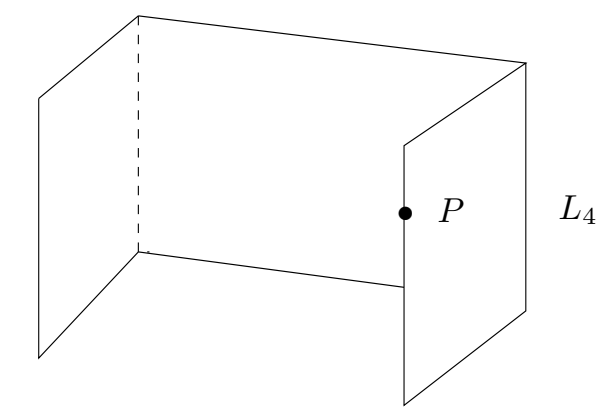

$\eta(P)=-\Delta_{2}$, and is zero elsewhere

Step 5 Calculate $\boldsymbol{\Delta} \eta \in \oplus \mathbf{E} \mathbf{x}^{2}\left(L_{i}\right)$. This is zero on all $L_{i}$ for $i \neq 4$, and equals $\Delta_{1} \wedge \Delta_{2}$ at the point $P$ in $L_{4}$ (see figure above).

- We can now see that $\Delta \eta$ is a restriction of a global 2 -form,

$$
\omega=\left\{\begin{array}{cl}
\Delta_{1} \wedge \Delta_{2} & \text { at } \mathrm{P} \\
0 & \text { elsewhere }
\end{array}\right.
$$

(Recall that restrictions on TopRight points of components are zero if forms of the appropriate dimension are not otherwise definable).

The overall result is,

$$
\begin{aligned}
& H_{\boldsymbol{\Delta}}^{2}\left(L S^{2}\right) \cong\langle\omega\rangle_{\mathbb{R}} \\
& H_{\boldsymbol{\Delta}}^{1}\left(L S^{2}\right)=0 \\
& H_{\boldsymbol{\Delta}}^{0}\left(L S^{2}\right) \cong \mathbb{R}
\end{aligned}
$$

where the zero-forms are the constant forms.

\section{Discussion, conjectures and open problems}

\subsection{Comparison with cubical cochain theories}

It is not hard to see that there is an injective map from the space of cubical complexes and their cochains to the space of lattice varieties and difference forms. For example, looking at the two lattice models in Example 20, it is clear that if one interpolates lines (1-cells) between adjacent vertices and 2-cells between adjacent lines, then one has two cubical simplicial models of the 2-sphere. Cubical chains are formal linear sums of cubical cells over some coefficient field, and cubical cochains are linear maps from the 
space of chains into the coefficient field. Thus a 2-cochain assigns a value to each 2-cell, a 1-cochain assigns a value to each edge and a 0 -chain assigns a value to each vertex. For each $p$-cell $\sigma$, with south west corner $v_{\sigma}$, we can construct a difference $p$-form from the cubical $p$-cochain $\omega$; the value of this $p$-form at $v_{\sigma}$ is $\omega(\sigma) \Delta_{i_{1}} \wedge \Delta_{i_{2}} \wedge \cdots \Delta_{i_{p}}$, where the $i_{j}$ label the coordinate directions of $\sigma$.

This injective map shows that there are no examples of discretized smooth spaces and forms that are missed by our theory. Moreover, this map commutes with the coboundary operators, so the cohomology groups are isomorphic.

However, the converse is false; not all difference forms can be obtained as the image of a cubical cochain. One can see this by noting that the image difference form has the property that every south west corner with $p$ adjacent lattice points in the direction of the relevant shift operators has a difference $p$-form defined on it. As Example 16 shows, this is false for lattice varieties in general. Lattice points may have difference forms defined on them up to the dimension of the variety, but they don't have to have them all.

We can summarize the discussion here and in the Introduction as follows.

- Lattice varieties need not be discretizations of a smooth space. There is no notion of an analogous tangent structure.

- The proofs and results in this paper do not assume that there exists a continuous space for which the lattice variety is a cubical chain approximation.

- The set of cubical cochains on a cubical complex maps injectively into the set of difference forms on lattice varieties, but not onto.

- There are analogues of exterior algebra, exterior derivative and Stokes' Theorem. The exterior difference (coboundary) operator does not satisfy the product rule (it is not a derivation) so proofs and constructions for difference forms cannot be inferred directly from those for smooth forms.

- Local potentials exists for the analogue of curl-free and divergence-free forms.

- Combinatorial notions such as the analogue of an open cover and a Čech complex on that cover can be used to study cohomology of difference forms on lattice varieties.

\subsection{Conjectures concerning discrete index theorems}

The Morse Index theorem relates the Euler characteristic of a manifold to features of the solution of a generic vector field on it, specifically to the indices of the vector field about its critical points (see for example [10], §14.3e). In this section we conjecture a result for generic linear equations on lattice varieties, relating the Euler characteristic of a lattice variety to an index (which we define) of the generic solution of the generic equation.

Suppose that you want to solve, globally, a 4-point scheme $\Omega$, defined locally as

$$
\Omega: \quad 0=a u_{n, m}+b u_{n+1, m}+c u_{n, m+1}+d u_{n+1, m+1}
$$


with $a b c d \neq 0$, on a 2-dimensional lattice variety. For simplicity, we consider only linear equations.

We demonstrate the kinds of calculations involved by considering solutions of (25) on the cube model of a sphere. In the diagram below, we have omitted the lattice points on the faces of the model, for simplicity.

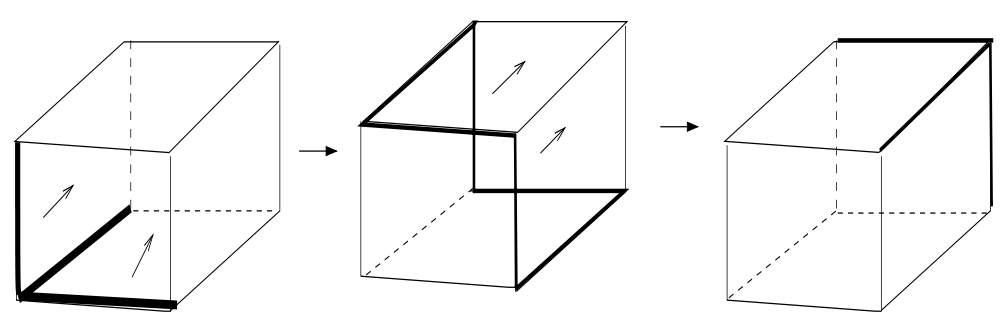

Begin with generic initial data defined on the points in the 1-dimensional sublattice variety shown as darkened lines in the left most sphere in the diagram. Then the equation can be solved at points in the bottom, left and front faces by sequentially solving for $u$ in the direction of the arrows on the left most sphere. This yields values for $u_{m, n}$ on the 1-dimensional sublattice (shown as darkened lines) of the middle sphere model in the figure. Continuing solving for $u$ (in the direction of the arrows drawn on the middle sphere) on the top, right and back faces, we obtain compatibility conditions on the initial data coming from the need to have $u$ well-defined on that sublattice of the third lattice model shown as darkened lines in the diagram.

A careful count reveals that regardless of the number of 2-cubes that make up the lattice sphere and regardless of the position of the initial data,

$$
\sharp \text { initial conditions }-\sharp \text { compatibility conditions } \equiv 2 \text {. }
$$

Conjecture 46 Let $L$ be a lattice model for a boundary-free manifold and let $\Omega=0$ be a generic linear equation defined on $L$. Define the index $\mathcal{S}(\Omega)$ to be

$$
\mathcal{S}(\Omega)=\sharp \text { initial conditions }-\sharp \text { compatibility conditions }
$$

for the generic solution, that is, the solution with arbitrary initial conditions. Then

$$
\mathcal{S}(\Omega)=\sum(-1)^{i} \operatorname{dim} H_{\Delta}^{i}(L) .
$$

We may take the right hand side of equation (26) to define the Euler Characteristic of $L$ by direct analogy with the result for smooth manifolds,

$$
\chi(M)=\sum(-1)^{i} \operatorname{dim} H_{\text {de }}^{i} \operatorname{Rham}(M) .
$$


We remark that the conjecture is not true for lattice models with a boundary, such as an annular lattice.

One can look at the Čech cohomology with local coefficients in the same way as for smooth manifolds, because the same constructions will hold (see [5], §10). If we take local coefficients on $L_{\boldsymbol{\alpha}}=L_{\alpha_{0} \cdots \alpha_{p}}$ to be the vector space $S_{\boldsymbol{\alpha}}$ of solutions of the linear equation $\Omega=0$ on $L_{\boldsymbol{\alpha}}$, then $S_{\boldsymbol{\alpha}}$ forms a pre-sheaf $\Sigma=\Sigma(\Omega)$ on $L$. Moreover,

$$
\begin{aligned}
\chi(L, \Sigma) & :=\sum(-1)^{i} \check{H}^{i}(L, \Sigma) \\
& =\sum(-1)^{i} \check{\mathcal{C}}^{i}(L, \Sigma) \\
& =\sum(-1)^{i}\left(\sum_{|\boldsymbol{\alpha}|=i} \operatorname{dim} S_{\boldsymbol{\alpha}}\right)
\end{aligned}
$$

follows from standard arguments.

Conjecture 47 Let $\Omega$ be a generic linear equation on a lattice model $L$ of a manifold perhaps with boundary. Then

$$
\chi(L, \Sigma(\Omega))=\mathcal{S}(\Omega) .
$$

\subsection{What is special about the south west?}

So far, the south west point of a fundamental $p$-cube is distinguished as the only point where a $p$-form is definable.

If instead of forward difference we want to use a backward difference or a consistent collocation, the entire theory above can be developed for different models of the basic fundamental cube. Simply, one fixes points at which the various forms are defined. In the diagram below, on the left is shown a fundamental 2-cube for a backward difference, while on the right an example is shown of a fundamental 2-cell for a collocation scheme.
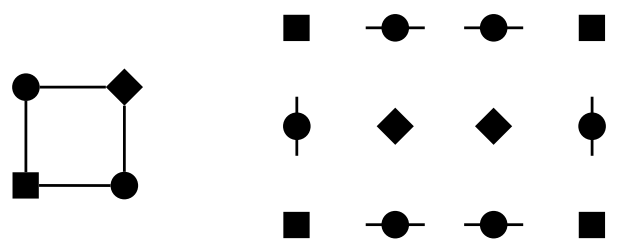

The right hand cell has no TopRight points, so the proofs for such a model are simpler than those we have presented. Gauss-Legendre, Marker and Cell and Preissmann schemes all have a fundamental model for what is defined where in a fundamental cube, and thus these schemes can be studied using the methods described.

\subsection{Open problems}

\subsubsection{Independence from the good cover}

In the smooth theory, one proves that the Čech forms are independent of the particular cover of the manifold used to calculate them by proving stability under refinement of 
the cover. As any two covers have a common refinement, independence follows. We do not have any such theory yet for lattice varieties. One conjecture would be that any two lattice models of the same manifold have the same cohomology, provided there are sufficient numbers of points.

\subsubsection{Non-orientable lattice varieties}

We leave open the problem of generalizing the methods used here to allow for lattice approximations of non-orientable manifolds. It is not hard to imagine a lattice approximation of a Möbius band, for example. They are excluded from the theory described here, as their construction necessarily violates the condition to respect the orientation inherited from $\mathbb{Z}^{p}$ when pieces of lattice are glued together to form a lattice variety. One interesting possibility is to find the analogue of a "twisted differential form" or pseudoform, see for example ( [10], §2.8).

\subsubsection{Localized refinements}

A common numerical technique is that of the localized refinement of a mesh. This is used to obtain more accurate information where the functions being calculated are rapidly changing. An open problem is how to adapt the constructions given in this article to cover meshes such as in the diagram. They are not covered by the theory described here as they violate the adjacency condition stipulated in the lattice variety construction.

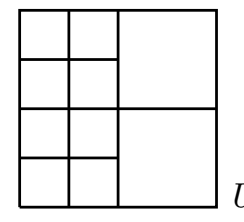

Using different scale meshes violates the adjacency condition.

\subsubsection{Functorial properties}

An open problem is to complete the difference form constructions described in this article to a fully functorial theory. This requires a discussion of mappings between lattice varieties, and of the associated maps they generate between cohomologies of the underlying spaces.

\section{Conclusions}

There is a strong analogy between global difference $(\boldsymbol{\Delta})$ forms of a lattice model of a manifold $M$, and smooth de Rham forms for $M$, by virtue of their relationship to a cover or decomposition of the underlying spaces. We have shown how to compute $\boldsymbol{\Delta}$ forms effectively. Our definitions do not rely on the existence of any underlying smooth space or continuous limit; therefore they make sense in the absence of any such model. 
The framework that we have described makes it possible to compute the cohomology of a given lattice variety. Consequently one can check whether or not the cohomology of a finite difference approximation is isomorphic to that of the continuous system that is being approximated. Furthermore, it is now possible to determine the cohomology of discrete systems that have no natural continuous limit. Many problems remain open, as described in $\S 6$. Our purpose in this paper has been to lay foundations.

\section{References}

[1] D. Arnold, Differential complexes and numerical stability, Plenary address at ICM Beijing, 2002. http://www.ima.umn.edu/〜arnold/papers/icm2002.pdf

$[2]$ D. Arnold, R. S. Falk and R. Winthur, Finite element exterior calculus, homological techniques and applications, Acta Numerica 15 (2006), 1-155.

[3] A. Bossavit, Differential forms and the computation of fields and forces in electromagnetism, Eur. J. Mech B-Fluids 10 (1991), 474-488.

[4] A. Bossavit, Whitney forms - a class of finite-elements for 3-dimensional computations in electromagnetism, IEE Proc-A 135 (1988), 493-500.

[5] R. Bott and L. W. Tu, Differential Forms in Algebraic Topology, Graduate Texts in Mathematics 82, Springer Verlag, New York, 1982.s

[6] C. J. Budd and G. J. Collins, An invariant moving mesh scheme for the nonlinear diffusion equation, App. Num. Math, 26 (1998), 23-29.

[7] C. J. Budd and M. D. Piggott, Geometric Integration and its Applications, Department of Mathematical Sciences Preprint 0024, University of Bath, 2000.

[8] J. A. Chard and V. Shapiro, A multivector data structure for differential forms and equations, Math. Comp. Sim. 54 (2000), 33-64.

[9] R. Forman, Morse theory for cell complexes, Topology 37 (1998), 945-979.

[10] T. Frankel, The Geometry of Physics, Cambridge University Press, Cambridge, 1997.

[11] W. Fulton, Algebraic Topology, A First Course, Graduate Texts in Mathematics 153, Springer Verlag, New York, 1995.

[12] Ge Zhong and J. E. Marsden, Lie-Poisson Hamilton-Jacobi theory and Lie-Poisson integrators, Phys. Lett. A 133 (1988), 134-139.

[13] P. W. Gross and P. R. Kotiuga, Electromagnetic Theory and Computation: A Topological Approach, MSRI Publications 48, Cambridge University Press, Cambridge, 2004.

[14] Gu Chaohao (Ed.), Soliton Theory and its Applications, Springer Verlag, Berlin, 1995.

[15] E. Hairer, C. Lubich and G. Wanner, Geometric Numerical Integration, Springer Series in Computational Mathematics 31, Springer Verlag, Berlin, 2002. 
[16] R. Hiptmair, Finite elements in computational electromagnetism, Acta Numerica 11 (2002),237-340.

[17] P. E. Hydon, Symmetries and first integrals of ordinary difference equations Proc. Roy. Soc. A 456 (2000), 2835-2855.

[18] P. E. Hydon, Conservation laws of partial difference equations with two independent variables, J. Phys. A: Math. Gen. 34 (2001), 10347-10355.

[19] P. E. Hydon and E. L. Mansfield, A variational complex for difference equations, J. Found. Comp. Math. 4 (2004), 187-217.

[20] A. Iserles, H. Munthe-Kaas, S. Norsett, and A. Zanna, Lie Group Methods, Acta Numerica 9 (2000) 215-365.

[21] T. Kaczynski, K. Mischaikow and M. Mrozek, Computing Homology, Homology, Homotopy and Applications 5 (2003), 233-256.

[22] L. Kharevych, W. Y. Tong, E. Kanso, J. E. Marsden, P. Schröder and M. Desbrun, Geometric, Variational Integrators for Computer Animation, in Eurographics/ACM SIGGRAPH Symposium on Computer Animation (M.-P. Cani nd J. OBrien eds.), ACM, Portland, 2006, 43-51

[23] T. Y. Kong, R. D. Kopperman, and P. R. Meyer, A topological approach to digital topology, Amer. Math. Monthly 98 (1991), 901-917.

[24] M. Leok, Foundations of Computational Geometric Mechanics, PhD Thesis, California Institute of Technology, 2004.

[25] R. I. McLachlan and G. R. W. Quispel, Six lectures on the geometric integration of ODEs, in Foundations of Computational Mathematics (R. A. DeVore, A. Iserles, and E. Süli, eds.), London Mathematical Society Lecture note Series 284, Cambridge University Press, Cambridge, 2001, pp. 155-210.

[26] E. L. Mansfield and P. E. Hydon, Towards approximations which preserve integrals, in Proc. ISSAC 2001 (B. Mourrain, ed.), ACM Publishing, 2001, pp. 217-222.

[27] W. S. Massey, Singular Homology Theory, Graduate Texts in Mathematics 70, Springer Verlag, Berlin, 1980.

[28] C. Mattiussi, An analysis of finite volume, finite element, and finite difference methods using some concepts from algebraic topology, J. Comp. Phys 133 (1997), 289-309.

[29] A. R. Mohebalhojeh, On shallow water potential vorticity inversion by Rossbynumber expansions, Q. J. Roy. Met. Soc., 128 (2002), 679-694.

[30] T. J. Bridges and S. Reich, Multi-symplectic integrators: numerical schemes for Hamiltonian PDEs that preserve symplecticity, Phys. Lett. A 284 (2001), 184-193.

[31] J. M. Sanz-Serna and M. P. Calvo, Numerical Hamiltonian Problems, Chapman and Hall, London, 1994.

[32] W. Schwalm, B. Moritz, M. Giona and M. Schwalm, Vector difference calculus for physical lattice models, Phys. Rev. E 59 (1999), 1217-1233. 
[33] S. Suuriniemi and L. Kettunen, Trade-off between information and complexity: a technique for automated topological computations, COMPEL 22 (2003), 481-494.

[34] Y. Y. Tong, S. Lombeya, A. N. Hirani and M. Desbrun, Discrete multiscale vector field decomposition, ACM Trans. Graphics, 22 (2003), 445-452.

[35] E. Tonti, On the formal structure of physical theories, Istituto di Matematica del Politecnico di Milano, Milan, 1975.

http://www.dic.univ.trieste.it/perspage/tonti/papers.htm

[36] A. Weil, Sur les théorèmes de De Rham, Comment. Math. Hel., 26 (1952), 119-145.

[37] H. Whitney, Geometric Integration Theory, Princeton University Press, Princeton, 1957.

[38] D. K. Wise, p-form electromagnetism on discrete space-times, Class. Quantum Grav. 23 (2006), 5129-5176. 